Elsevier required licence: (C) <2021>. This manuscript version is made available under the CC-BY-NCND 4.0 license http://creativecommons.org/licenses/by-nc-nd/4.0/

The definitive publisher version is available online at

[https://www.sciencedirect.com/science/article/pii/S0001868621000038?via\%3Dihub] 


\title{
Janus membranes for membrane distillation: Recent advances and
}

\section{challenges}

\author{
Morteza Afsari, Ho Kyong Shon, Leonard D. Tijing* \\ ${ }^{\mathrm{a} C e n t r e ~ f o r ~ T e c h n o l o g y ~ i n ~ W a t e r ~ a n d ~ W a s t e w a t e r, ~ S c h o o l ~ o f ~ C i v i l ~ a n d ~ E n v i r o n m e n t a l ~ E n g i n e e r i n g, ~}$ \\ University of Technology Sydney, P. O. Box 123, 15 Broadway, NSW 2007, Australia \\ *Corresponding author: L.D. Tijing, email: leonard.tijing@uts.edu.au
}

\section{Abstract:}

Membrane distillation (MD) is a promising hybrid thermal-membrane separation technology that can efficiently produce freshwater from seawater or contaminated wastewater. However, the relatively low flux and the presence of fouling or wetting agents in feed solution negate the applicability of MD for long term operation. In recent years, 'two-faced' membranes or Janus membranes have shown promising potential to decrease wetting and fouling problem of common MD system as well as enhance the flux performance. In this review, a comprehensive study was performed to investigate the various fabrication, modification, and novel design processes to prepare Janus membranes and discuss their performance in desalination and wastewater treatment utilizing MD. The promising potential, challenges and future prospects relating to the design and use of Janus membranes for MD are also tackled in this review.

Keywords: Janus membrane; membrane distillation; desalination; wastewater treatment; membrane fabrication 
2. Overview and design of Janus membranes

2.1. Anti-fouling and anti-wetting properties

3. Janus membrane fabrication methods 15

3.1. Hydrophilic on top of hydrophobic configuration ... 16

3.2 Hydrophobic on top of hydrophilic configuration ........................................................ 26

3.2.1 Liquid-liquid interface .26



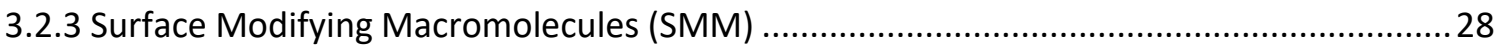

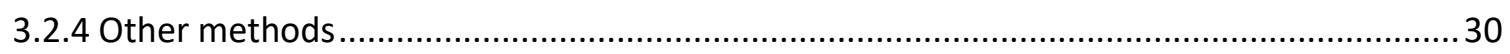





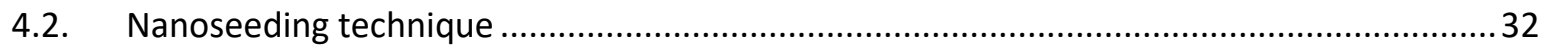

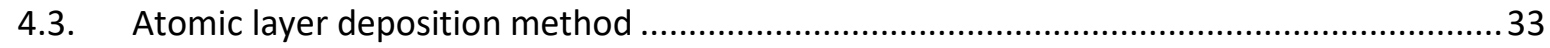



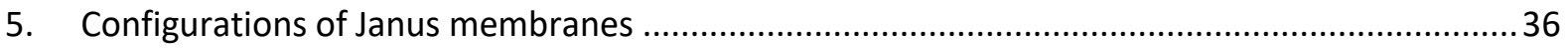

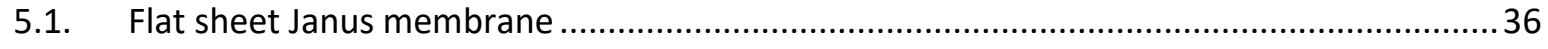

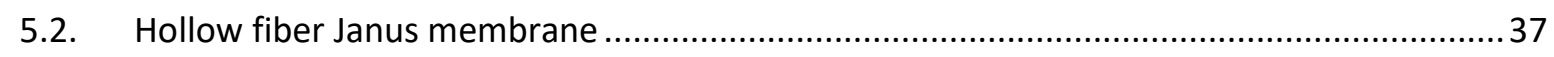

5.3. Electrospun Janus nanofiber membrane ............................................................ 43

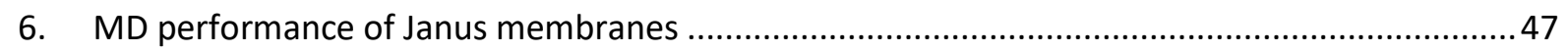

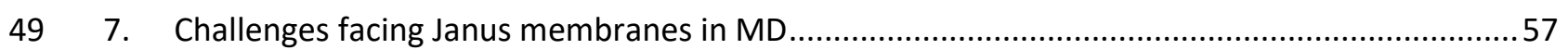

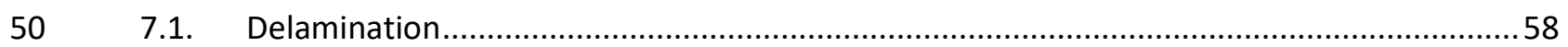

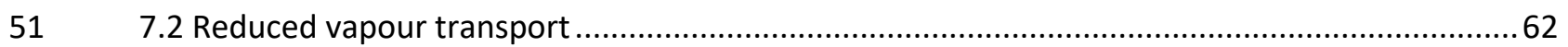

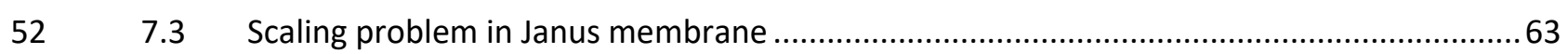

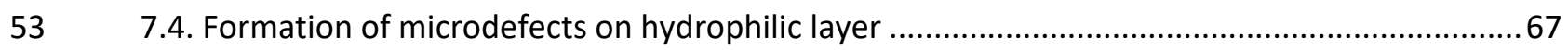

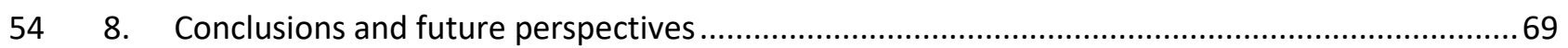

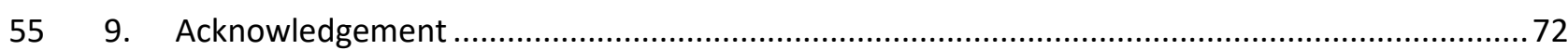

List of abbreviations. 


\section{Introduction}

Global water scarcity, driven by rapid urbanization, population growth and climate change, is

a critical issue nowadays and is expected to get worse in the next decade. As a result, alternative sources of fresh water such as from seawater and wastewater are being sought out [1]. The vast amounts of seawater available makes desalination as a viable option for freshwater extraction. However, the state-of-the-art reverse osmosis (RO) that is widely used in many countries, still has limitations in treating high salinity brine. In recent years, membrane distillation (MD) process has driven increased interest due to its ability to treat hypersaline solutions, challenging wastewaters [2] and even for resource recovery [3]. MD is a thermal-based membrane separation system which benefits from possible use of low-grade thermal energy and is a brilliant candidate to treat a wide range of water sources from common brackish and seawater to hypersaline RO retentate, shale-gas or coal-steam gas produced water, and highly polluted wastewaters [4, 5]. Though MD has relatively lower energy efficiency compared to RO [6], it still has some advantages over RO in terms of its ability to utilize low-grade heat sources such as waste heat and solar energy, is highly suited for modular system configuration, and can treat hypersaline solutions that is above the salinity limit of RO [7]. Despite these advantages, industrial application of MD is still limited due to low flux and wetting issues of currently used membranes. Wetting happens when liquid water overcomes the entry pressure of the membrane pores starting from the largest pore size, thus penetrating and reaching the permeate side. Wetting is exacerbated when 
dealing with challenging wastewaters that contain inorganic salts, humic acid and low surface tension components such as surfactants, oils, etc.

Although different studies generally attributed the wetting mechanism to the change in hydrophilicity of membrane pores by adsorption of the surfactants on the pore walls (Figure 1) [8], recent studies focusing on the wetting mechanism proved that the main reason for pore wetting is the reduction of surface tension of the feed stream [9]. These studies also proved that adsorption of the surfactants on the pore walls can delay the wetting by removing the surfactants from the water-gas frontier [9-11].



Figure 1 Pore wetting schematic in a microporous membrane [10]

Many recent studies have tried on increasing the hydrophobicity of the membranes by increasing the surface roughness. The rough surfaces can trap the air and decrease the slipping angle of the membrane, resulting to higher hydrophobicity. The strategies include nanoparticle (NP) coating or incorporation [12, 13] such as Si [12], Al [14], and Ti [15] NPs, coextrusion [16, 17], co-spinning, electrospraying [18], spray-assisted non-solvent induced phase separation [19], grafting [20], and phase inversion techniques [21, 22]. Though the superhydrophobic membranes improved their wetting resistivity, however, their oleophilic characteristics can still lower the durability of the membranes, and fouling is still a drastic 
problem [23-25]. To address this drawback, omniphobic membranes, which can repel both

water and low surface tension agents have been designed and investigated [26]. This can be obtained by designing a hierarchical structure or re-entrant surface structure followed by coating with a low surface energy layer $[27,28]$. However, omniphobic membranes are still prone to fouling issues due to hydrophobic foulants like oil droplets [11,29]. Thus, continued efforts are still done to design a membrane that can potentially reduce fouling formation, while resisting wetting and maintaining adequate flux and rejection.

Inspired from nature, especially from sea species like clamshell and sharkskin, many groups have worked towards designing underwater oleophobic surfaces which can dramatically decrease fouling problems made by microorganism or by organic fouling for MD application. Among the various suggested methods, the Janus membrane (multilayer hydrophilic/superhydrophobic or hydrophilic/omniphobic membrane) or 'two-faced' membrane (i.e., both sides of the membrane have different wetting properties) is a promising structure for MD systems for the treatment of challenging wastewaters from food processing, leather and fabric industries, shale gas well drilling, and domestic sewage [30, 31]. For MD application, a Janus membrane, which has one side hydrophilic layer and one side omniphobic or hydrophobic layer can potentially repel most of fouling agents like oil particles [18, 22, 27]. The hydrophilic layer repels the oil droplets and other hydrophobic compounds and prevent their adhesion on the surface, and the hydrophobic or omniphobic base layer mitigates the wetting problem $[28,32]$.

Additionally, to enhance the driving force in MD and consequently have high permeate flux, membranes with low mass transfer resistance and high heat transfer resistance are favourable. The preferred membrane for MD process to have high flux is a porous and thin 
membrane. However, this feature can also dramatically decrease the strength of the membrane, especially if used in vacuum MD (VMD) modules, and can increase the heat loss through the membrane. As a result, to overcome this problem, the best suggested method is fabrication of Janus membrane to increase the thickness of the membrane (by adding hydrophilic layer) without increment in hydrophobic thickness [44]. Studies have reported that Janus membranes can enhance the flux performance and decrease the heat loss, while reducing the propensity for wetting and fouling $[27,33]$. The hydrophilic layer of the Janus membranes plays as a fouling barrier. In addition, it works as a heat barrier and decreases the total heat transfer without sacrificing the mass transfer coefficient $[1,41]$.

This review presents the recent advances progress on the fabrication, challenges and application of Janus membranes for MD [27, 34-36]. To the authors' knowledge, no review has been carried out so far focusing only on Janus membranes for MD. We start our discussion with the fundamentals of Janus membrane design, and then present some innovative strategies for Janus membrane fabrication and modification. The application and evaluation of Janus membrane are also elaborated and finally, the effect of Janus membranes in overcoming fouling and wetting problems as well as flux improvement is thoroughly elucidated.

\section{Overview and design of Janus membranes}

Janus is the name of the ancient Roman god that has two opposite faces; one looks to the past and the other looks to the future [37, 38]. In material science field, Janus was firstly used by De Gennes for synthesis of particles that chemically have different hemispheres [39]. The 

materials. Accordingly, materials with ambivalent properties is called Janus, like Janus particles, Janus nanosheets, and Janus membrane. Janus membrane was firstly introduced by Cheng and Wiersma in 1982 and since then the number of research focused on improvement methods of Janus membrane drastically increased [40]. Janus membrane is a new configuration that has asymmetric wettability in both sides. In other words, one side is hydrophilic and the other side is hydrophobic or omniphobic [41]. Figure 2 shows a schematic of the various designs of Janus membranes. As shown in this figure, the Janus membrane can be fabricated by coating of hydrophilic layer on top of a hydrophobic, superhydrophobic, or omniphobic base membrane. The fabricated Janus membrane can be used in both



Hydrophobic Membrane



Janus membrane on Hydrophobic Membrane

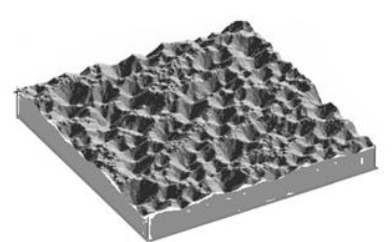

Superhydrophobic Membrane

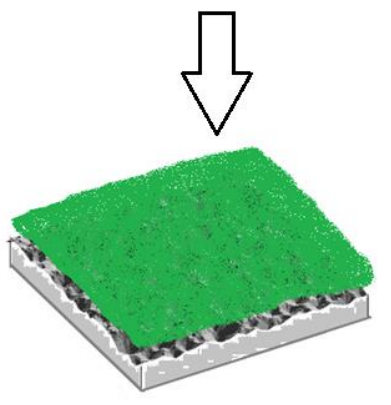

Janus membrane on Superhydrophobic Membrane

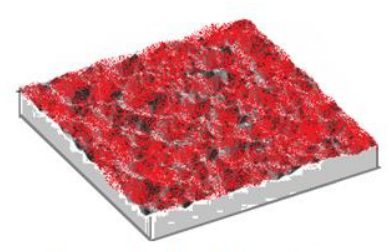

Omniphobic Membrane

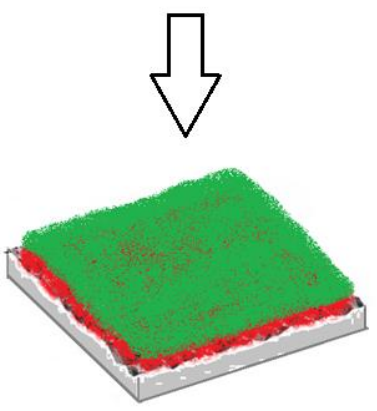

Janus membrane on Omniphobic Membrane

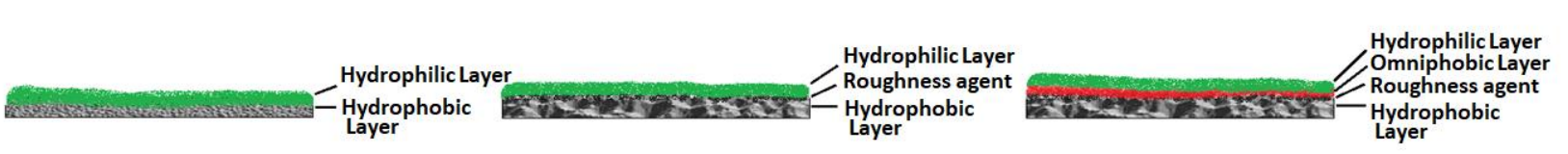


According to the mass and heat transfer conflicts in MD systems, the optimum thickness can be approximately calculated using the pore size and heat conductivity coefficient. For Janus membrane, the optimum thickness can also be designed accordingly. Reports indicated that the optimum thickness of the hydrophobic part (with porosity $>70 \%$, thermal conductivity $=$ 0.1-0.3 W/mK) is in the range of $30-60 \mu \mathrm{m}$ [16][69]. However, most of hydrophobic membranes have been fabricated having the thickness of around $100-150 \mu \mathrm{m}$ to compensate its low mechanical strength [69].

Hydrophobicity or hydrophilicity is dependent on both the morphology and surface energy of the material. These parameters determine the tendency of the surface to adhere or repel the liquid materials. The wetting properties are dependent to the chemical structure of the surface and also to the roughness, pore size, and environmental condition. Empirical correlations can be used to predict the surface affinities of various liquids, but the easiest and straightforward evaluation method is via measurement of liquid contact angle. In general, surfaces with water contact angle (WCA) greater than $90^{\circ}$ are considered hydrophobic and less than $90^{\circ}$ are hydrophilic [4]. Different liquid types will have varying affinity to a particular material and surface. For membranes having rough and porous surfaces, the contact angle can be estimated using Wenzel equation for homogenous and Cassie-Baxter for heterogeneous types. Figure 3 shows the transition lines for different wetting states versus the roughness of the surface [42]. In addition, Figure $3 \mathrm{~b}$ depicts the state of the water droplet

174 in both Wenzel state and Cassie-Baxter state [43]. 
(a)



(b)

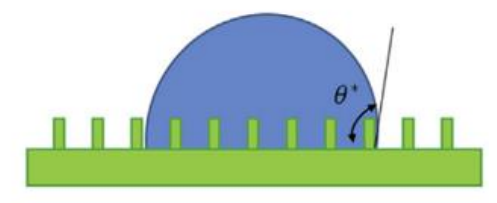

Wenzel state

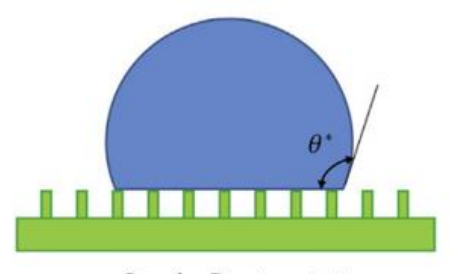

Cassie-Baxter state

Figure 3. (a)The transition line from Cassie-Baxter regime to Wenzel regime [42], and (b) the state of droplets in both Wenzel andCassie-Baxter states [43].

According to the physical and chemical properties of the surface and liquid, different states from Wenzel state to metastable and stable Cassie-Baxter state are formed. The Cassie-Baxter state shows the low interaction of liquid with the membrane surface that results to higher hydrophobicity and lower slippery angle of the membrane, which are suitable for MD application. In this condition, upward capillary forces inhibit intrusion of liquid into the grooves of membrane surface and preserve the membrane from wetting [44, 45].

\subsection{Anti-fouling and anti-wetting properties}

Janus membranes, which possess opposite wettability at two sides of the membrane can lead distinct surface energy provides a specific wettability condition for Janus membranes [46]. 
When exposed to different types of liquids including water, mineral oil, ethanol, or surfactantrich water, Janus membranes can potentially limit their wicking into the membrane compared to other membranes. For example, superhydrophobic membranes are adequately resistant to high surface tension liquids but are easily wetted by low surface tension liquids $[47,48]$. Omniphobic membranes perhaps are highly regarded for MD processes, as they possess good resistivity against surfactants and wetting agents, but still suffers from fouling issue in oilpolluted wastewaters $[27,32]$. High volume of oil-containing wastewater produced during different food and shale gas drilling process make it crucial to find an effective and low cost process for oil water separation $[49,50]$. Oil droplets in feed can lead to quick fouling of the membrane surface, resulting to decrease in flux, clogging and wetting issues that affect its utilizing the positive value of superhydrophobic or omniphobic substrates to deal with these issues.

Several studies have shown that hydrophobic and omniphobic membranes have less resistance to underwater oil droplet due to the hydrophobic-hydrophobic interaction between oil droplet and the membrane surface. In the case of hydrophobic membrane, due to the strong hydrophobic-hydrophobic interaction, oil wicks through the membrane, clogs the pores and causes fouling of the membrane [29]. For omniphobic membrane, the membrane surface is also covered by the oil particles due to hydrophobic interaction and foul the membrane, however the oil particles do not wick into the pores and the fouling is reversible via commonly used cleaning methods like backwashing [11]. Janus membranes have thin hydrophilic layer that are hydrated with water, showing underwater oleophobicity, which can repel oil droplets and avoid oil fouling formation. Furthermore, according to 
an increase in the area of hydrated top layer and consequently enhances its oleophobicity.

This was proven by study of Huang et al, wherein the hydrophilic layer of the Janus membrane was coated with silica and chitosan to increase both the wettability and surface roughness of membrane. This led to the reduction of fouling generated by oil droplets on the Janus membrane [48, 51]. Another study also demonstrated the effect of hydration of the hydrophilic layer on repulsion of oil droplets from depositing on the surface [34, 52]. Wang et al. [52] are the pioneers of using force spectroscopy to analyse the oil fouling of membrane. In their work, the adhesive force between oil droplet and membrane surface for the hydrated top layer of Janus membrane was less than $0.02 \mathrm{mN}$, while that of hydrophobic PVDF membrane reached to around $0.2 \mathrm{mN}$ (see Figure 4). This proportion shows relatively high repulsive force of Janus membrane for oil droplets. The receding curve also shows dramatic decrease for hydrophobic membrane (from about $0.18 \mathrm{mN}$ to less than $0.08 \mathrm{mN}$ ) which reveals the presence of high adhesive interaction. In other words, the hydrophobic membrane attracted the oil droplets at the time of contact and showed higher possibility of fouling, whereas the change in adhesive force for Janus membrane is zero, demonstrating that no interaction occurred during the contact and detachment $[29,52]$. In addition, DCMD test results revealed higher fouling resistivity of Janus membranes compared to hydrophobic or omniphobic membranes. 


\section{(A)}

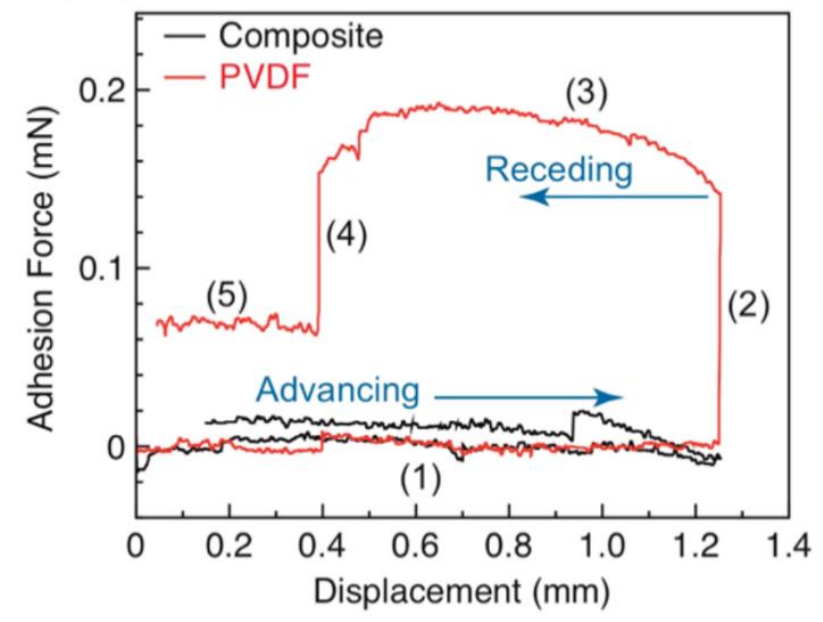

(B)
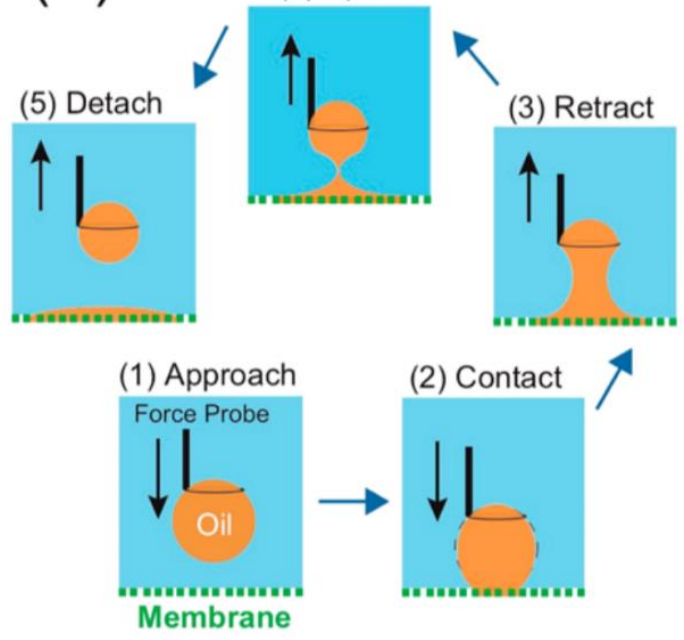

Figure 4. Force-distance curve for dynamic movement of oil droplet into contact of both Janus and hydrophobic membrane through advancing and receding movement [52].

In another study, the wettability performance of Janus membrane prepared by coating of PDA on omniphobic PTFE/PP-Teflon was compared with hydrophobic PTFE-PP membrane, under various types of liquids. Results showed high interaction of hydrophobic membrane with low surface tension liquids but low interaction on Janus membranes. This low interaction is attributed to the presence of omniphobic membrane substrate beneath the thin hydrophilic layer repelling the low surface tension liquids in the process. This exceptional behaviour helped the Janus membrane to work for long term MD operation with stable flux and salt rejection, while other tested membranes showed reduction in performance after some hours of testing with contaminated and polluted feed water [53]. Li et al. also investigated the effect of coating of hydrophilic layer on the wettability of prepared membranes [27]. The results proved that Janus membrane enjoyed a near perfect salt rejection and constant flux during the treatment of seawater containing all types of wetting and foulant agents. This study also demonstrated that the thickness of the hydrophilic layer can have dramatic effect on fouling 
and wetting resistance of omniphobic membrane, so care must be taken to come up with the optimum thickness [27].

Zhu et al. fabricated a Janus membrane by coating hydrophilic PAN layer on F-SiO 2 @PVDFHFP/PS omniphobic membrane. The hydrophilic PAN (4 wt\%) solution was coated on the omniphobic membrane by electrospinning, and the formed structure resulted to a dramatic increase in underwater OCA to more than $164^{\circ}$, proving underwater superoleophobicity of Janus membrane [33]. Long term MD operation of the Janus membrane demonstrated a stable flux of $25 \mathrm{LMH}$ and $100 \%$ salt rejection for $50 \mathrm{~h}$ continuous test, while hydrophobic PVDF and superhydrophobic PVDF NFM showed quick fouling after only half hour of test. The dynamic study of the fouling mechanism showed that oil droplets attached and fouled first on the other two membranes, but could not attach on hydrophilic PAN layer of Janus membrane. The unattached oil droplets were then aggregated on the surface of Janus membrane and formed bigger oil droplets and then left the Janus membrane surface without any fouling problem $[33,54]$. The dynamic investigation of wetting and contact angle can helpful to assess the DCMD experimental results.

The effectiveness of Janus membrane for increasing the resistivity of membrane against fouling and wetting problems also depends on the composition of contaminants in the feed water. The structure and features of surfactants can change the fouling mechanism in the Janus membranes. The ionic surfactants can adsorb on the membrane surface via the electrostatic or hydrophobic forces. Although the negative charge of hydrophobic membrane (like PVDF membrane) repels the negative side of surfactants, however, it can interact with the other side of the surfactant and adsorb it. Therefore, the flux reduction in hydrophobic 
membrane could be attributed to this interaction. In this interaction, the hydrophobic to

hydrophilic ratio of surfactants can determine the power of hydrophobic-hydrophobic interaction of membrane and surfactants and show stronger adsorption of surfactants on the membrane $[55,56]$. However, the adsorption rate is determined by the diffusion, which is irrelevant to the strength of the adsorption. In other words, smaller hydrophilic-lipophilic balance (HLB) of surfactant represents stronger adsorption, but does not show faster adsorption and does not demonstrate the higher probability of wetting issue in the MD process [56]. For Janus membranes, the hydrophobic and electrostatic interactions play the most important roles. For example, in the case of PDA-PEI/PVDF Janus membrane, the protonated amine functional group can make electrostatic interaction by sulfate groups of hydrophilic parts of surfactants and induce fouling for the membrane $[18,22,27]$.

On the other hand, when Janus membrane deals with cationic surfactants like DTAB, the positive charges of protoned amine-functional groups in the membrane and quaternary ammonium heads of the DTAB repel each other and the intrinsic structure of Janus membrane keeps the membrane surface from surfactant fouling. For the case of hydrophobic membrane, like PVDF, the negative charge of the membrane surface can attract the positive parts of DTAB and make both electrostatic and hydrophobic interaction and make wetting problem for hydrophobic membrane. In one fouling study, the surfactant-stabilized oil in water emulsion was prepared as the fouling agent [57]. The results revealed that the oil-water emulsion has positive charge and is repelled by positive charge of protonated amine-functional groups on the Janus membranes and helps the hydrogen-bond in hydrated layer for prevention of oil fouling on the Janus membranes (as depicted in Figure 5) $[57,58]$. However, when the feed water contains free surfactant in addition to surfactant-stabilized oil, the free surfactants easily pass the hydrophilic layer of the Janus membrane and make wetting beneath the 
hydrophobic layer [29]. In general, the effectiveness of a modification should be investigated case by case and the moieties of the feed water and membrane structure should be recognised.


Oil $\}$ DTAB

$\mathrm{O} / \mathrm{W}$ emulsion

Figure 5. the anti-fouling procedure of feed water containing surfactant-stabilized oil in Janus membrane [57]

\section{Janus membrane fabrication methods}

Depending on the face of the Janus membrane that is on the feed side, the application of the Janus membrane varies. In general, in relation to the wettability characteristics of the top face of the membrane, the Janus membrane is fabricated for two general applications: lowering the fouling or lowering the mass transfer resistance. In former state, the hydrophilic layer is in contact with feed stream and the Janus membrane is used for antifouling application (i.e, it can repel the foulant). In latter case, the hydrophilic layer of Janus membrane is in contact with permeate side and Janus membrane is used for increasing the mass transfer of the MD process, without sacrificing the thickness of the membrane that affect its mechanical stability. The thickness and other features of hydrophilic and hydrophobic layer varies, depending on the application of Janus membrane. In this way, the Janus membrane is fabricated using two general methods: deposition of hydrophilic layer on top of hydrophobic membrane or vice 
versa. In this section, the fabrication process of Janus membrane is categorised according to the base substrate: incorporation of hydrophilic layer on top of a hydrophobic/omniphobic substrate or incorporation of hydrophobic layer on top of a hydrophilic substrate.

\subsection{Hydrophilic on top of hydrophobic configuration}

The most common method for the fabrication of Janus membrane, which have been widely used in many studies, is by deposition or incorporation of a hydrophilic layer on top of a hydrophobic or omniphobic membrane substrate [36]. This method has the advantage of being generally simple, straightforward, and is a step-wise process [59]. However, the modification methods in many cases compromise the quality of the substrate membrane by clogging the pores or changing the hydrophobicity of the membrane. Furthermore, stepwise preparation increases the material and fabrication costs and increases the delamination problems $[37,60]$. Fabrication of new membranes is usually done by phase inversion, hollow fiber spinning or by electrospinning. However, these processes in many cases do not directly create the Janus membrane structure, thus further modification processes are required. The modification processes include coating, incorporation of nanoparticles or surface modifying macromolecules $(\mathrm{SMM})$, electrospinning or electrospraying of environmental friendly hydrophilic materials like PEG, PDA, hydrogels, grafting of hydrophilic functional groups, and other ways of providing specific wettability and function to the membrane [61-63]. In other studies, before modification of the membrane surface, some chemical methods such as plasma treatment were used to prepare the hydrophobic substrate to be more affinitive to the hydrophilic layer. This section presents the various ways to fabricate and modify Janus membranes with hydrophilic-hydrophobic structure. 


\subsubsection{Vacuum filtration}

Vacuum filtration is a simple and straightforward method for coating of a hydrophilic top layer on hydrophobic or omniphobic base membrane. In this method, firstly a hydrophobic or omniphobic microporous flat sheet membrane is fabricated and then a hydrophilic layer is coated on top layer using vacuum filtration of a solution containing desired hydrophilic nanoparticles (NPs). The size, dimension, and chemical structure of NPs are very important parameters in defining the efficiency of the Janus membrane. However, this method suffers from low stability and delamination problem. For example, one study covered the top layer of a PVDF membrane by vacuum filtration of solution containing Si NPs which resulted to an increase in membrane surface roughness [64], while another study added CNT containing solutions which led to a decrease in roughness for the same type of substrate [65-67]. The explanation for this modification can be attributed to the size and shape of the nanoparticles and also the presence of ridge-valley structure on the PVDF membrane surface. This condition can cause a decrease in porosity, which can led to diminished flux performance [34]. Even though the CNT containing membrane decreased the pore size distribution of the Janus membrane by blocking or decreasing the effective area of some pores, its mechanical stability compared to unmodified membrane has increased. TGA data showed higher thermal stability and mechanical analysis of modified membrane revealed that imposing strains of PVDF-CNT membrane was about two-fold compared to unmodified PVDF membrane. However, the tensile strength obtained similar results to that of neat membrane [34]. The inorganic nature of CNTs and also the functional groups present on its surface can have interaction with the substrate and other CNTs to make a strong deposited layer, having sufficient hydrophilic wettability $[60,68]$. Generally, the physical and chemical structure of CNTs make possible rapid mass transfer of water molecules through outer and inner surface of CNTs via 
sequential sorption-desorption and can increase water transport to the membrane surface and simultaneously make a barrier against oil droplets for some special wastewater treatments like oil-emulsion treatment [34].

\subsubsection{Coating via co-casting phase inversion}

Co-casting is another method wherein both layers are subsequently casted and can be used to fabricate bilayer or multilayer materials, such as Janus membranes. One important factor in the investigation of the effect of simple coating procedure is considering the structure of layers during coating. In phase inversion, the type of polymer, solvent, nonsolvent, and temperature are important factors of layering. The structure and morphology of casted solution is formed according to the miscibility of the solvent and nonsolvent and the difference in chemical and physical properties of matrix polymer may cause delamination during coagulation process. Therefore, the thermodynamic properties of different mixtures should be considered to find optimum type of solvent and nonsolvent $[61,69,70]$. The ternary phase diagram should be used to determine the miscibility of polymers, solvents, and nonsolvent to estimate the optimum condition to fabricate integrated multi-layer membrane. For example, in Figure 6, the black line shows the binodal curve of PVDF dope solutions containing three compositions (polymer, solvent, and non-solvent). As indicated in this figure, addition of PEG or silica nanoparticles to dope solution changed the phase diagram of dope solution by moving the binodal curves and showed a decrease in power of the solvent in mixed matrix polymer solution versus neat dope solution. [71] 


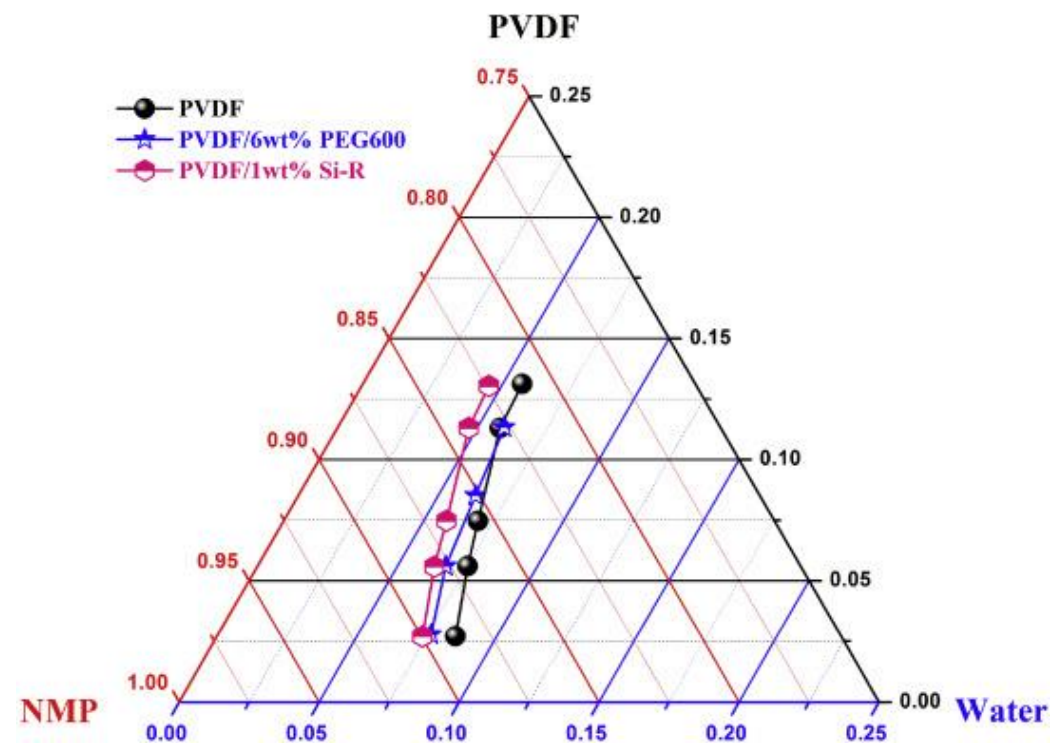

Figure 6. The Ternary diagram for coagulation of different types of PVDF/NMP solution in water bath [71].

384 In another study, in the process of co-casting of PVDF-PVA as hydrophilic layer and PVDF as hydrophobic beneath layer, due to difference in solvent replacement, two distinct layers were 386 formed (Figure 7). Difference in solvents and polymers resulted in the fabrication of asymmetric membrane with an obvious boundary between two layers. The difference in rate 388 of miscibility of solvents in two phases into nonsolvent coagulant bath formed two distinct layers which increased the possibility of delamination [69]. Depending on the type of phase inversion process (TIPS or NIPS), the coagulation process has direct influence on the structure of the Janus membrane $[22,72,73]$. 


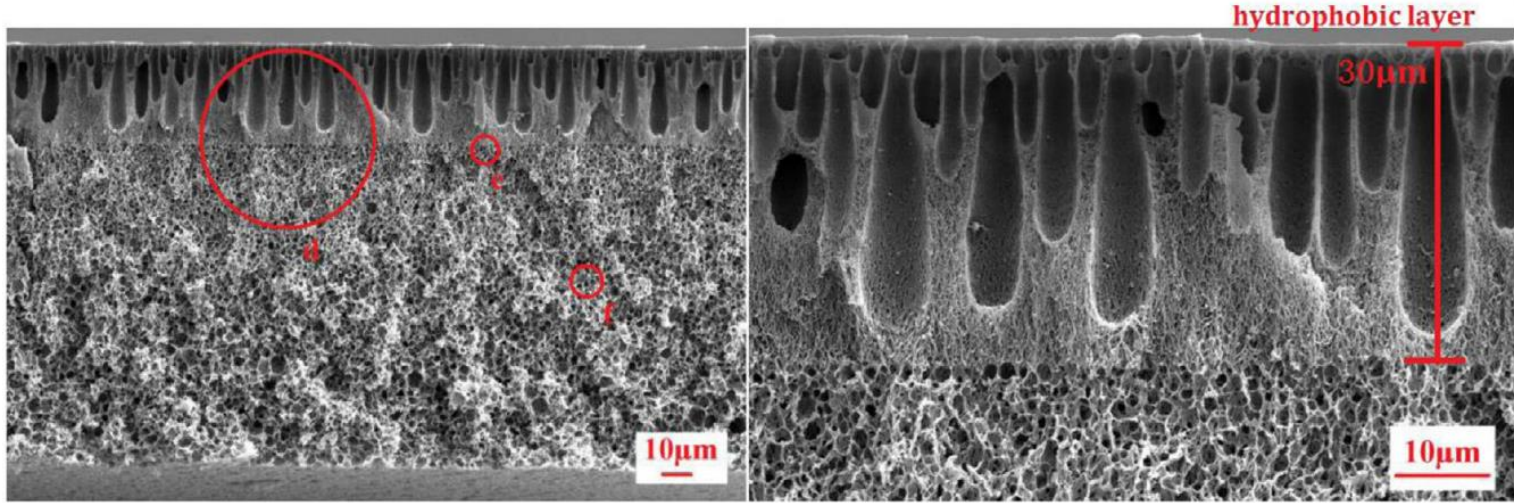

Figure 7. Cross section SEM image of flat sheet bilayer membrane fabricated by PVDF-PVA

on PVDF [69]

\subsubsection{Asymmetric fabrication}

Asymmetric fabrication refers to the one-step fabrication of Janus membranes as opposed to

symmetric fabrication where a hydrophilic layer is subsequently coated on a hydrophobic or

omniphobic layer. In the asymmetric method, usually the difference in solubility of materials

is used to fabricate asymmetric structure. In brief, a dope solution containing at least two

distinct types of materials, like polymers or nanoparticles, is prepared and the dope solution

401

is placed in a coagulant environment. Then the asymmetric structures start to form due to

402

difference in surface energy of materials and their tendency to reach the low energy surface.

403

Also in some cases, the difference in solubility of polymer composite in a coagulant liquid causes faster migration of polymer parts toward the outer surface and causes fabrication of a membrane with different wettability in both sides $[30,33,74]$.

Additionally, it is possible to fabricate Janus membranes by internal migration of materials through the membrane matrix and make a polar hydrophilic structure. In this method, usually a dope solution containing both hydrophilic and hydrophobic materials is prepared and then the solution placed in an asymmetric surface energy environment. In this condition, the 
macromolecules or monomers, which are not yet coagulated, tend to migrate to surfaces with lower energy, according to their chemical affinities. This movement usually occurs in phase inversion process. In this process, the nonsolvent tends to remove solvents and replace them, according to solvent-polymer-nonsolvent interaction in ternary phase diagram. In a heterogeneous polymer solution containing both hydrophilic and hydrophobic polymers, the polymer parts tend to migrate to the more-soluble nonsolvent, due to their intrinsic interactions. For example, if the water is nonsolvent, the hydrophilic parts tend to migrate toward the outer surface and, as a result, can change the hydrophilicity of outer layers. In this method asymmetric membrane can be fabricated without delamination problem. For comparison, in a normal phase inversion, both top and bottom layer become similar in hydrophilicity, due to simultaneous migration of hydrophilic part of solution towards all sides. In order to preserve the asymmetric style, novel methods like adhesion of an impermeable layer to one side of the casting surface should be used $[22,61]$. Li et al. utilized glycerol to coat a nonwoven fabric, then a polymeric solution is casted on the fabric and then immersed in coagulation bath. As a result, a solution containing copolymer PVP-VTES and PVDF was prepared. The PVP-VTES has hydrophilic affinity and tends to have interaction with water to reach lower surface energy. Compared to PVDF, the PVP-VTES had faster migration rate. The presence of glycerol layer in the bottom side of the membrane makes a barrier against the solvent-nonsolvent exchange and diminishes the phase separation on the beneath layer. Therefore, the hydrophilic copolymer cannot penetrate across the bottom layer of the membrane and the migration occurs only in one side of the casted layer and the difference in miscibility rate results to an asymmetric hydrophilic-hydrophobic structure [22]. various difficulties like wetting of the substrate pores via hydrophilic layer. MD membranes 
have microporous structures and the pores usually can be wetted due to capillary force and interactions between the bulk membrane and coating layer. As a result, if the modification method does not perform accurately, not only that the wetting and fouling not hindered, but also the separation performance of the hydrophobic substrate decreases. Some methods have been suggested to enhance the modification process. One of these methods include placing a separation interface to prevent the modification layer from intruding into the pores of the substrate. In this method, usually a thin layer is coated on the surface of the hydrophobic membrane and then a hydrophilic layer is entangled with this intermediate top layer and two distinct regions are formed. The intermediate layer plays the role of making strong adhesion on both hydrophilic and hydrophobic layers and also prevents the pore clogging by the hydrophilic layer. In another type of method, it is possible to adjust the reaction time of the polymeric dope solution to make a gradient reaction across the membrane width. In this method the composition of dope solution is accurately selected to have different reaction rate and difference in reaction rate make integrated asymmetric membrane with opposite wettability in both sides $[31,68,75,76]$.

\subsubsection{Two-phase interface method}

450 One of the difficulties of Janus membrane fabrication is modification of only one side of the substrate. It is not so straightforward to modify only one side of a polymeric surface having only some micron thickness. Therefore, an applicable modification method should have capability of changing the wettability of one side of the membrane without changing the characteristics of the other side. The dilemma is the selection of proper solution. A wetting solution may intrude into the pores and decrease the efficiency of the MD process, while a non-wetting solution may not interact with the membrane substrate and may delaminate the 
top layer. Various methods have been proposed to modify the surface of hydrophobic membrane. Two phase interface method is a novel coating method that can be used to modify only one side of the membrane, without affecting the other side. In this method the membrane substrate is soaked in the interface of two immiscible liquids, so that one side of the membrane is in contact with the surface of the other liquid. Then the desired layer is reacted with the membrane substrate to form a layer on top of it $[22,59,77]$. For instance, Yang et al. have floated PP membrane on a solution containing PDA/PEI. This resulted to a hydrophilic layer of PDA/PEI coated on the hydrophobic PP membrane and a Janus membrane was fabricated. In this method, it is essential to coat the membrane surface with high viscosity solution to prevent intrusion of solution into the substrate pores via capillary force [77]. Similar method also can be applied by restricting one side of membrane substrate by sticking an impermeable layer and then soaking the membrane inside a reaction liquid. In this way, the solution can react with only one side of the membrane and a hydrophilic layer is coated on the membrane substrate. The sticked layer preserves the other side of the substrate from solution reactants. After finishing the reaction, the impermeable coating is peeled-off and Janus membrane is fabricated. However, the peeling-off step in most of cases destroys the beneath layer of the Janus membrane and may affect the performance of the membrane for application in DCMD $[60,78]$.

\subsubsection{UV-mediated modification strategy}

Another method for the fabrication of Janus membrane is treating one side of the membrane using photoreaction or photoresist materials. In this method, the membrane substrate can be soaked in a photoreacted or photoresist solution and then one side of the membrane is activated by irradiation. After the reaction is carried out, the remained photoresist or 
photoreacted material is removed and a Janus membrane is fabricated. However, care must be taken to make sure that the photosensitive or photoreacted material must not affect the wettability of the membrane substrate after removal from the membrane surface.

UV-sensitive reactions are also good way to the modify one side of the membranes. Thiol-ene click reaction is an example of reaction that is activated by UV-light. This method has benefits of rapid reaction rate, high yield, easy process, and controllable directional reaction. Li et al modified different types of substrates by firstly coating of PDA, using mussel-inspired catechol chemistry, and then silanized it to produce superhydrophobic substrates. The polycondensation of Trichlorovinylsilane provides photosensitive functional groups. Afterwards, the superhydrophobic substrate is immersed in thiol-ene click reaction solution and was irradiated by UV-light, which renders one-side of the membrane surface hydrophilic.

This modification process makes a membrane having asymmetric wettability with $140^{\circ}$ WCA difference between two sides of the membrane. According to the pore size distribution and thickness of the substrate, this membrane can be used for different water treatment applications like MD, oil-in-water emulsion, or water-in-oil emulsion separation [80].

\subsubsection{Multi-step coating method}

Wang et al. fabricated a Janus membrane using a multistep method to coat hydrophilic polyamine in one side and a hydrophobic polymer at the other side. In this study, cotton fabric was deposited by a compound of PDMS containing light sensitive materials. Then one side of deposited cotton was irradiated to crosslink the light sensitive material and make a strong connection with the fibers. Afterward, the remaining deposited material was washed using hot THF solvent. Then, the other side of the fiber was grafted by propyl methacrylate groups, using sol-gel method and a catalytic reaction, to increase the hydrophilicity of Janus 
membrane. Therefore, hydrophilic cotton fibers become hydrophobic/superhydrophilic Janus membrane after these consecutive process[81] . Furthermore, it is possible to combine multieffect of coated layer to enhance effectiveness of prepared Janus membrane for some special applications, like incorporation of nanoparticles on top of hydrophilic layer of Janus membrane to cover its microdefects. However, due to change in structure of hydrophilic layer during hydration process, the stability of coated layer is very challenging and its stability should be investigated. In order to assess the stability and stiffness of the coated layer, especially for nanoparticle coating, the first step is to use an ultrasonic instrument. In this method, the loose bonding is detected and the applicability of the Janus membrane for long term operation is determined [57]. In this way, it is possible to assess the stability of coated nanoparticles by placing the used membrane in high frequency ultrasonic bath to determine the percentage of nanoparticles that remained on the surface of the Janus membrane [32, 82].

Generally, the surface energy of PVDF is higher than PTFE, but it has been widely used for MD process due to its good compatibility with different polymers and solvents, low cost, and adequate mechanical stability. Therefore, a wide range of polymers that are compatible with PVDF are used for the fabrication of bilayer or blended Janus membrane. In order to decrease delamination, it is better to choose polymers with close solubility parameters to make interactions during coating process. Although PVDF has been widely used as hydrophobic substrate for MD process, it can be modified to become hydrophilic or superhydrophilic substrate. In other words, its useful properties can be used in other side of technology to have high water wettability characteristics [24, 83-86]. In a study, Zhou et al. fabricated a Janus membrane by changing a hydrophobic PVDF membrane to superhydrophilic membrane by initiation of vinyltriethoxysilane cross-linking reaction that converted it into a 
superhydrophilic PVDF substrate. The superhydrophilic PVDF was casted on a PET nonwoven fabric and after coagulation, it was peeled off to generate micro and nano size rough structures and then superhydrophobic fluorinated silica nanoparticle solution was sprayed on these torn surface to coat the substrate and make a superhydrophobic layer. Regarding to the degree of superhydrophobicity of the top layer, this Janus membrane can be used in different methods of water treatment, like forward osmosis or MD [87].

\subsection{Hydrophobic on top of hydrophilic configuration}

Although most of the Janus membranes are fabricated by coating of hydrophilic layer on top of hydrophobic or superhydrophobic membrane, it is possible to do the opposite fabrication method: coating of hydrophobic layer on top of hydrophilic layer [93]. The following are different methods used for this type of fabrication method.

\subsubsection{Liquid-liquid interface}

In a novel method, liquid-liquid interface was used for the incorporation of a hydrophobic layer on top of a hydrophilic substrate. For this purpose, firstly the hydrophilic cotton membrane was soaked in dopamine solution for deposition of intermediate layer. Then the DA-coated cotton membrane was floated on the water - dichloroethane containing octadecylamine $\left(\mathrm{C}_{18} \mathrm{NH}_{2}\right)$ two phase beaker. The membrane is placed at the liquid-liquid interface, according to a range of densities. The amine groups of $\mathrm{C}_{18}-\mathrm{NH}_{2}$ has hydrophilic tendency and stay orientated toward water interface. Therefore, this intrinsic property caused interaction of amine group with dopamine molecules deposited on the surface of cotton membrane. The deposition of PDA on the hydrophilic cotton fiber prepared the medium for attachment of $\mathrm{C}_{18}-\mathrm{NH}_{2}$ hydrophobic layers. The water-oil interface prepares the 
exchanging area for this attachment. After interaction, the prepared Janus membrane has a

550 relatively thin hydrophobic $\mathrm{C}_{18}-\mathrm{NH}_{2}$ layer beneath a thick hydrophilic layer [35]. However, this

551 technique is only possible to perform on flat sheet membranes. In another study, Vanagamudi

552 et al. have fabricated Janus membrane by casting of hydrophobic PVDF layer on the

553 electrospun nanofibers made of hydrophilic nylon/chitosan blend. The presence of hydroxyl

554 and amine functional groups give high hydrophilicity to the electrospun nanofiber. Although

555 the Janus membrane showed reduced pore size, the flux and rejection increased compared

556 to neat PVDF membrane [88].

\subsubsection{Electrospinning deposition}

558 Yan et al. have used a novel method by deposition of hydrophobic electrospun nanofiber on superhydrophilic non-polymeric substrate. The substrate was constructed from porous copper mesh having nanosize needles. This substrate was prepared by immersion of smooth copper mesh into $\mathrm{NaOH}$ solution having $\left(\mathrm{NH}_{4}\right)_{2} \mathrm{~S}_{2} \mathrm{O}_{8}[89,90]$. This process changed the wettability of the substrate from hydrophobic to superhydrophilic feature (WCA changes from $114^{\circ}$ to $\left.0^{\circ}\right)$. The nanosized needles can intrude into the deposited nanofibers and enhance coating interaction and decrease the possibility of delamination and, in general, increased the entanglement of deposited hydrophobic layer with the substrate. Furthermore, the formed interface roughness increases the hydrophobicity and hydrophilicity of Janus membrane. The top layer was deposited using electrospinning of polymer solution containing different PVDF concentration. The change in the concentration of the PVDF solution, changed the properties of nanofibers. While beads were formed on the nanofibers fabricated at lower concentration, the beads disappeared at higher PVDF concentration. In addition, Tijing et al.

571 have fabricated a dual-layer membrane for DCMD application by electrospinning of PVDF-HFP 
on PAN microfibers. In this method, the PAN nenofibers firstly was electrospun on the drum and then the PVD-HFP nanofibers was electrospun on the PAN substrate. The prepared membrane showed superb porosity of $90 \%$ and WCA of $150^{\circ}$ at the feed side and complete salt rejection, completely suitable for DCMD application [91].

\subsubsection{Surface Modifying Macromolecules (SMM)}

In general, Janus membranes can have two configurations: asymmetric wettability in a distinct layer or having gradient wettability from top to bottom layer. The former type has a separating layer that connects the hydrophilic and hydrophobic layers together. The latter usually is formed by migration of materials having opposite wettability through the dope solution during coagulation or processing time and the concentration gradient makes the wettability gradient across the membrane. Application of surface modifying macromolecules (SMM) in the fabrication of Janus membrane provides a gradient change in wettability across the membrane $[92,93]$. SMMs are a group of active additives that tend to move toward the lower surface energy surfaces and can migrate in non-solidified phase to reach to lower interfacial energy. In other words, the SMMs in a dope solution have the ability to move to all-sideward to reach the surfaces and to have minimum interfacial energy. The small percentage of SMMs are sufficient to make a heterogeneous layer. Therefore, the application of SMMs has shown great potential for the fabrication of Janus membrane, especially for integrated membrane for MD application. The promising point of SMM is about the possibility of one step fabrication, which decreases the commercial costs. SMMs are usually fluorinated polymer segments produced by fluorination of polymers like polyurethane, PVDF, and PES and are dissolved in a solution containing hydrophilic polymer solution. The dope solution is casted and placed in an air environment phase inversion process. The waiting time lets the 
SMMs to migrate toward the surface having low surface energy and make an asymmetric Janus membrane with a gradient wettability: hydrophobic top layer and hydrophilic bottom layer [94]. The type of blended polymer, SMM polymer and fluorocarbon, and dope solution solvent are important parameters affecting the mechanical, physical, and chemical properties of Janus membrane. Zhang et al. reported the use of different types of solvents like $\mathrm{CHCl}_{3}$, $\mathrm{CH}_{3} \mathrm{CN}$, THF and acetone for the fabrication of SMM-based Janus membrane. Their results indicated that the best condition was achieved by using $\mathrm{CH}_{3} \mathrm{CN}$ solvent achieving the highest wettability difference between the two sides $[90,94]$.

The incorporation of SMM in hydrophilic membranes increases the chemical, mechanical, and thermal stability of the membranes and covers the delamination drawbacks for application of Janus membrane in different wastewater treatment application. One of the most important challenges is due to the all-directional movement of SMMs in dope solution, while the preferred movement direction is only one side migration. The derived membrane after phase inversion process usually have hydrophobic surface and hydrophilic bulk. In order to efficiently use the migration behaviour of SMM, the fabrication process needs to be modified using methods for controlling the directional migration. The covering of one side of the membrane is a possible method that have been used to direct the SMMs toward only one surface [22]. Before focusing on application of SMMs in Janus membrane, SMMs were used for enhancement of the hydrophobicity of the base membrane. In some research, hydrophilic PEI membrane was modified with SMM to fabricate Janus membranes for MD application [94, 95]. Also, in a series of studies performed by Khayet's group, fluorinated SMMs were blended with hydrophilic PEI to enhance the LEP of the membrane $[17,96]$. 
618 Other researchers also have used hydrophilic porous substrate like cellulose acetate or 619 cellulose nitrate and coated hydrophobic layers like styrene or vinyltrimethylsilicon compounds to fabricate bilayer Janus membranes. However, these researches have used

621 radiation graft or plasma polymerization methods that are relatively complicated and expensive for large scale fabrication [16, 97]. Also, most of studies were performed in flat sheet module and more research on other commercial modules like hollow fiber is necessary. content of fluorine in its structure. The fluorine content increases the chemical and thermal resistivity of the polymers and form a superhydrophobic compound. Beside this outstanding properties, fabrication of porous PTFE membrane is so complicated and expensive. On the other side, PVDF, a fluorine-containing polymer that are being used as MD membrane, has lower hydrophobicity compared to PTFE. Therefore, applying novel methods to use the benefits of PTFE and decreasing the complicity and cost of process for MD application is favourable [2-4]. In a study performed by Figoli et al, a UV-sensitive PTFE layer was coated on commercial hydrophilic polyamide membrane. In this work, commercial microfiltration PI membrane was dip coated in PTFE oligomer solution and then one side of coated layer was cured by UV light. The UV-process stabilized PTFE on one side of the PI membrane and the remaining PTFE from other side was washed out to produce a Janus membrane. The derived membrane had high hydrophobicity, owing to the PTFE layer, and was fabricated simpler than commercial PTFE microporous membranes. Additionally, it had the advantages of being a Janus membrane [98]. 
641 Gas plasma technology is a chemical-energy modification method, which changes the 642 structure of the material to the phase other than three regular solid, liquid, and gas phases. In this technology, usually high voltage is applied to the materials to ionize them and the materials are brought to the plasma phase. In this phase, a controlled reaction on a narrow and thin layer can be performed, according to the properties of used gas and also substrate surface. Plasma technology is widely used in membrane technology to change the properties of the surface layer to desired property, mostly to prepare surface for adopting of a coating layer [26]. Plasma etching can scratch a very thin layer of the membrane substrate and prepare condition for making interaction with coating layer $[99,100]$.

For a Janus membrane, the membrane substrate has hydrophobic or omniphobic properties and the top layer has hydrophilic wettability. The opposite wettability characteristics of these layers make challenges in the adhesion process. Therefore, gas plasma method can be used to ionise the hydrophobic or omniphobic substrate surface and change the structure for hydrophilic attachment. For example, Li et al. treated the surface of PP substrate via plasma for two minutes (at $200 \mathrm{~W}$ ) to coat a hydrophilic top layer. The treated surface effectively accepted the coating of a Teflon layer for increasing hydrophobicity of the substrate and also attachment of hydrophilic PDA layer [53]. Zuo et al. have used plasma technology to modify the surface of PEG and $\mathrm{TiO}_{2}$-coated PVDF membrane. The etching technique modified the surface of a hydrophobic PVDF membrane to graft with PEG functional groups. The FTIR spectrum showed a decrease in both asymmetrical and symmetrical stretching bond of $\mathrm{CF}_{2}$ at 
successful modification process. This grafting process caused changing in the wettability of the membrane to hydrophilic and fabrication of Janus membrane. However, the grafting approximately halved the average pore size of the membrane, which is beneficial for decreasing the wettability, but also decreases the flux of the membrane.

In another study performed by Lee et al, a Janus membrane was successfully fabricated by soaking of porous hydrophilic alumina substrate in photoresist AZ 5214 and then etching one side of the membrane by air plasma. Afterwards, the etched surface was silanized using low surface energy perfluorodecyltrichlorosilane to make an omniphobic thin layer. The presence of photoresisted material makes a barrier against infiltration of the membrane pores with silane groups preserving its hydrophilicity during the modification process. Afterwards, the remaining photoresist was washed out and removed. This fabrication procedure produced a Janus membrane with top hydrophobic coating and bottom substrate hydrophilic structure. This novel method also can be used for other modification techniques. After etching one side of the membrane, it is possible to coat-etch the surface of the substrate by other polymeric hydrophobic layer using methods like vapour deposition or interfacial layer deposition [101].

\subsection{Nanoseeding technique}

Nanoseeding is a novel technology for changing the structure of the surface by increasing the surface roughness and its physical and chemical characteristics. In this method, first, the nanoseeds are stabilized on the substrate surface and then the nanorods are grown on the activated sites of the substrate. This modification method also can be used to increase the hydrophobicity of the substrate by growing of hydrophobic nanorods. Furthermore, due to barbed morphology of the surface, it is possible to immobilize a layer containing opposite wettability to fabricate Janus membrane. The combination of bumped rods with chemical 
interaction provided by thermal and mechanical treatment can fix the top layer on the superhydrophobic substrate $[102,103]$. In a study, ZnO nanorods were grown on a hydrophilic cellulose acetate fiber in arrays and then superhydrophobic layer was made by immersion in sodium laurate solution. Afterwards, hydrophilic $\mathrm{MnO}_{2}$ nanowires were coated on one side of the substrate via vacuum filtration and hydrothermal treatment. The modified membrane has shown superb properties like high porosity, asymmetric wettability and highly stabilized coated layer. The substrate showed superhydrophobic characteristic with WCA of $153^{\circ}$ and sliding angle of $3^{\circ}$. However, the OCA of the substrate is $0^{\circ}$ that shows oleophilicity of the substrate, which indicates that it is susceptible to rapid fouling. The asymmetric modification process makes a surface oleophobic and substrate hydrophobic that provides condition for treatment of foulant-rich contaminated wastewater, using MD method. Additionally, the stability of the coated layer was tested by immersion of the membrane in a hot water/ethanol solution. This solution swells both layers and provide maximum layer stress. The results showed no detachment of $\mathrm{MnO}_{2}$ nanowires that prove high interaction of coated layer [97].

\subsection{Atomic layer deposition method}

Layering technology also can be used to fabricate a Janus membrane via atomic layer deposition (ALD) method (see Figure 8). ALD is a precise technique used in semiconductor fabrication process for layer by layer conformal growth. In this method, a metal oxide layer having molecular-sized thickness is deposited on desired surface without changing the structure of the pores. Therefore, this method can be used to precisely coat an oxide metal having hydrophilic features on the surfaces of hydrophobic or superhydrophobic substrate. Waldman et al. have modified the surface of hydrophobic PP membrane by deposition of hydrophilic aluminium oxides to fabricate Janus membranes. The results demonstrated that 
Janus membrane having high wettability difference was fabricated without considerable change in porosity and pore size distribution of the substrate. Also, it was revealed that the coating depth and degree of hydrophilicity can be tuned by controlling exposure dose and time of the process. Also, molecular precision of ALD makes it possible to provide sharp wettability difference across a narrow line in one side of the substrate. [36]
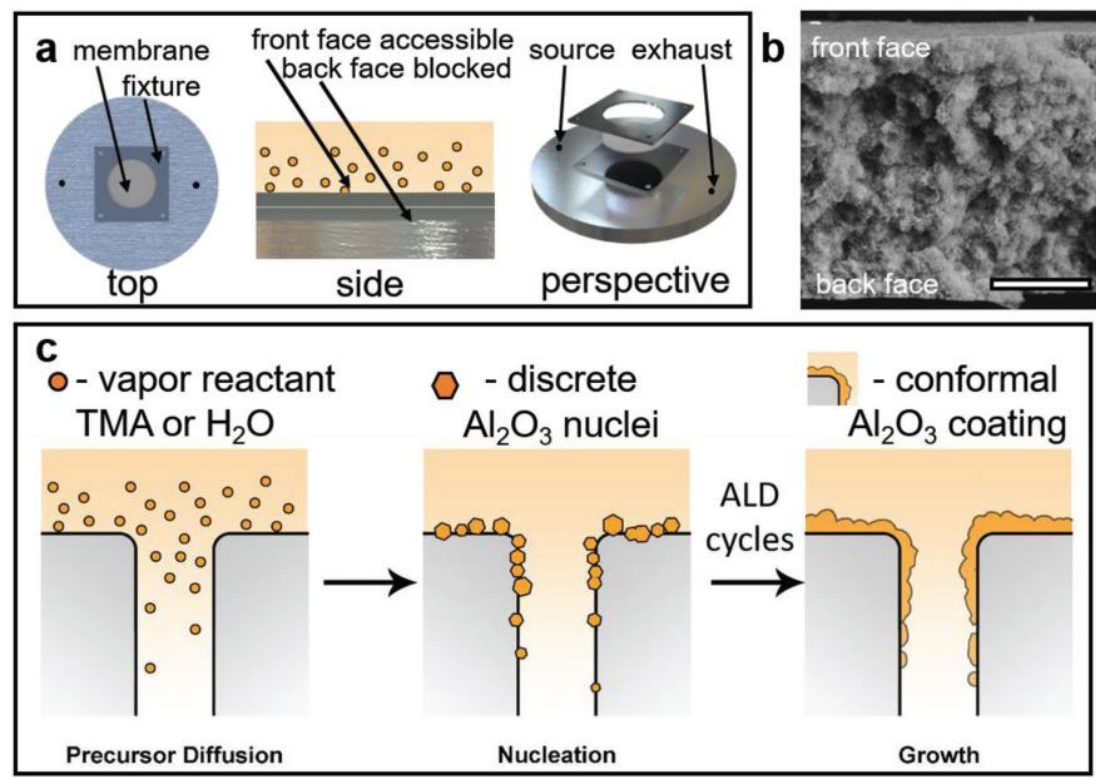

Figure 8. Atomic Layering Deposition Technique used for changing the characteristics of top Layer [36]

\subsection{Other methods}

Other novel methods like laser modification are also possible for surface modification and fabrication of Janus membrane. However, these methods are expensive and time consuming and only has justification in some special applications. In this method, both sides of hydrophilic substrate are roughened and coated with low surface energy material to produce 
superhydrophobic membrane. Then, one side of the surface is treated with laser to scratch fluorinated or low surface energy coating [75].

In one study, Ghaleni et al. modified PVDF flat sheet membrane with concentrated $\mathrm{KOH}$ to graft hydrophilic functional groups on the membrane surface. During the reaction, the high alkaline condition breaks the carbon-hydrogen and carbon-fluorine bonds and generate carbon hydroxyl bonds. The zeta potential analysis performed on the modified membrane showed that the surface became more negatively charged, compared to unmodified membrane, owing to its hydrophilic structure that adsorbs more anions like $\mathrm{OH}$ - or $\mathrm{Cl}$ - from the electrolyte [61]. Some studies also used facile fabrication of Janus membrane by simply putting hydrophobic mesh filters on top of hydrophilic substrates, usually hydrophilic fabrics like cotton. This method can be used for special applications like fog harvesting, but due to low adhesion interaction and also large pore sizes of hydrophobic meshes cannot be used for applications like MD or oil-water separation [31]. Also, it is possible to premodify the membrane surface to increase the efficiency of prepared Janus membrane. For instance, The Si nanoparticles, which have been widely used to increase the roughness of the membrane, has negative charges and in the membrane surface should have positive charge to make possibility of interaction of Si NPs with membrane surface. Therefore, charge modifiers like TEOS or CTAB can be used to change the charge of membrane surface to positive. After deposition of roughening materials like silica nanoparticles, the surface of rough membrane is coated with low surface energy coating like fluorine materials to make in air omniphobic membrane. Now, the hydrophilic layer like composition of $\mathrm{Si}$ NP/chitosan/ perfluorooctanoate can be coated to make a superb Janus membranes. If the attachment process is carried out perfectly, a high performance Janus membrane is achieved with mentioned layer characteristic [48]. 
Layer-by-layer assembly is another technique for modifying the surface of hydrophobic membrane for fabrication to Janus membrane. In this method, one side of the hydrophobic membrane (like PVDF) is sealed and then the membrane is periodically immersed in various solutions containing intermediate and hydrophilic solutions. This subsequent immersions is termed as layer-by-layer coagulation of dope solutions [104].

\section{Configurations of Janus membranes}

\subsection{Flat sheet Janus membrane}

The easiest structure to prepare for MD application is flat sheet membranes and they have been widely fabricated to investigate the performance of various Janus membranes [68]. In a study, the effect of changing the solvent of PVDF-based membrane on delamination was investigated by fabrication of flat sheet Janus membrane [69]. This simple membrane structure besides ease of production process provides a condition for fast analysis of the effect of various parameters on the property of bi-layer Janus membrane. Li et al. also fabricated a flat sheet Janus membrane comprising of an omniphobic layer composed of PVDF-silica NPs coated with FDTES low surface energy as substrate and hydrophilic layer by coating of atom-transfer radical-polymerization (ATRP) on the plasma-etched substrate. The flat sheet structures helped in the investigation of the effect of low thickness coating of hydrophilic layer on both foulant and wetting resistivity of prepared Janus membrane. Also, the effect of operating condition on flux and salt rejection was easily studied [27].

In general, the layer deposition is the simplest modification method for fabrication of Janus membrane. In this method, which is commonly applicable for flat sheet membranes, a thin layer of desired coating solution is coated on the surface of the substrate. Due to low viscosity 
of the solution at the time of casting, pore wetting may be an issue for this method. Also, high viscosity solution may clog the pores of the membrane. The interaction of coating layer with the membrane substrate also is another important factor that affects the performance of the membrane for long term operation. In a study, the glutaraldehyde-PVA solution was prepared, coated, and incubated on the surface of PVDF membrane. The substrate pores were preserved from wetting by controlling the physical condition of the solution. The derived flat sheet membrane showed good coating with low pore wetting and high water productivity [105]. In some studies, before coating of the main hydrophilic layer, the top surface of the membrane is pre-treated to prepare conditions for strong interaction of hydrophilic layer with the substrate. Plasma etching or coating with intermediate polymer solutions like PDA is some examples of this intervention. Although some of these methods showed superb layer interactions and great separation performance, the applicability of suggested method for large scale and commercial system is not satisfied. In general, flat sheet membrane is generally thought as not a favourable module for industrial application. Furthermore, multicommercialization potential[105].

\subsection{Hollow fiber Janus membrane}

One of the most optimum modules for commercialization of water treatment application is hollow fiber membranes. This structure has relatively high aspect ratio and low facility volume per volume of produced permeate water. Although its fabrication in lab scale is more difficult than flat sheet membranes, it is favourable for industrial and large-scale production. Therefore, the most facile and straightforward modification methods should be applied on hollow fiber membranes to find shortcut path for commercialization [106]. A negative point 
of the hollow fiber membranes is having high-pressure drop along the membrane that increases the working pressure of membrane and as a result, the wetting possibility of the membrane. Furthermore, due to exponential correlation of vapour pressure with temperature, temperature decrease in the module and as a result, the flux dramatically decreases in the end of the membrane. Therefore, the optimum condition is to have a low length hollow fiber membrane with feed inlet through the shell side with higher contact surface area and permeate gathered through the lumen side of the membrane $[16,107-110]$.

Regarding high promising potential of hollow fiber membrane for wastewater treatment applications, different studies have focused to modify or fabricate hollow fiber Janus membrane. Various types of methods have been proposed including vapour deposition, lumen coating, outer surface coating, and co-extrusion. Among these methods, coating process is simplest method, but the homogeneity and lamination are two main problems during fabrication and operation of this type of Janus membrane. The one-step fabrication of Janus membrane is desirable to simultaneously save the time and cost. For this purpose, it is possible to use triple orifice spinneret to co-extrusion of two polymeric solution with opposite wettability characteristics. From large-scale production point of view, the coextrusion is most efficient and applicable method for production of membrane modules. With respect to other modification methods, co-extrusion method decreases the possibility of delamination of distinct layers. However, the proper selection of dope solution and coagulant bath condition are crucial section of production process. The type and category of solvents used for preparation of both dope solutions and their interaction during extrusion and coagulation are determining the quality of the hollow fibers and the possibility of delamination. Therefore, the co-extrusion has shown great flexibility through dope preparation and fabrication process $[17,96]$. Zou et al. fabricated Janus hallow fiber 
membrane by co-extrusion of PVDF/PEG and PVDF Si-R dope solution and NMP/water as bore fluid. One advantage of this method is the use of NMP as solvent for both dope solutions. Therefore, the unique solvent helped the preparation of integrated membrane without separable layer. The presence of Si nanoparticles also increased the roughness of the outer hydrophobic layer and PEG increased the hydrophilicity of inner layer. As depicted in Figure 9, the composition difference make two different morphologies for inner and outer part of the membrane; PVDF-Si NP formed a thin hydrophobic outer surface with WCA of $137^{\circ}$ and PVDF-PEG formed thick hydrophilic inner layer with WCA of $56^{\circ}$ [108]. This difference can be attributed to the difference in inner and outer coagulation bath condition and difference in dissolving rate of polymers in coagulant solution. The presence of NMP in the bore fluid decreases the exchanging rate of solvent-nonsolvent and produce porous structure with small pore sizes, whereas the pure water coagulant bath in outer surface prepared high dissolution rate and finger-like structure during phase inversion process is formed. The superhydrophobicity properties of Si-R was also effective on formation of such configuration, which prevent extensive distribution of water molecules through dope solution for NMPwater exchanging. As a result, instead of liquid-liquid mixing, solid-liquid mixing occurred and porous and triangle structure is formed. The interface of two dope solution is obvious in the SEM images (Fig 7), however the similar solvent make strong uniformity between two phases and decreased the possibility of delamination.

Although these studies proved the benefits of co-extrusion process, it has some limitation in the selection of polymer and solvent and the tolerance in degree of hydrophilicity and hydrophobicity of Janus membrane layers. In other words, co-extrusion method compromises the wettability difference between top and bottom layers for overcoming of lamination challenge. 



Figure 9. SEM images of Janus membrane fabricated using co-extrusion technique $(a, b)$ and the inner and outer morphology and their surface WCA $(c, d, e)[108]$

842 The surface modification of hollow fiber membrane is another attractive method for modification of currently industrialised hollow fiber membranes. The advantage of this method is on the modification of a standard hollow fiber membrane that have uniform and applicable substrate structure. However, the drawbacks of coating procedures are still challenging for this modification process. One of the commonly used procedure is using mussel-inspired technique to adhere hydrophilic layer in inner or outer surface of hydrophobic hollow fiber membrane. However, the modification of lumen is more straightforward. For example, Yang et al. have modified the lumen side of PP hollow fiber membrane by coating of PDA/PEI dope solution. In this method, the dope solution is stepwise intruded through the pores of the substrate and changed the wettability of inner layer to hydrophilic ones. The thickness of hydrophilic layer can be adjusted by changing deposition time. The presence of dopamine in the coating dope made a tough and strong hydrophobic 
adhesion by substrate and a uniform structure was formed. The depth of penetration and

thickness of hydrophilic layer of Janus membrane is measured by using EDX scan. PDA can chelate silver ions and make bond via its catechol groups. Therefore, Ag solution was circulated through the lumen of hollow fiber and after chelating, EDX scan is taken from the cross section of membrane to evaluate the terrace of silver ions, which is equivalent to thickness of hydrophilic layer. The results showed that about $20 \%$ of thickness of hydrophobic membrane was coated by hydrophilic layer (about $90 \mu \mathrm{m}$ out of $450 \mu \mathrm{m}$ ) [107]. In another study, Zuo et al. have focused to fabricate a Janus hollow fiber membrane by co-extrusion of PVDF and Ultem dope solutions. The bilayer Janus membrane showed more than four times higher tensile strength and high flux, compare to conventional one-layer PVDF membrane [111]. Polyetherimide also is a good substrate for dual layer Janus membrane fabrication for MD application, owing to its mechanical strength, hydrophilicity, and compatibility with PVDF. Even though the Polyetherimide Ultem is immiscible with PVDF, the solubility parameters of both are so close, which can make tough molecular interaction between two connected layers.

Another important point in the fabrication of bilayer Janus membrane is the concentration of polymers in dope solution. Regarding to the fact that after coating process, the dope solutions start to shrink, the relative concentration of inner and outer layer has direct influence on the quality of interface layer. In hollow fiber membranes, the shrinkage direction is towards the centre. Therefore, if the concentration of outer layer is less than theinner layer, the shrinkage process causes the outer polymer solution to be firm around the lumen polymer solution. This transition makes a tough and tight interface layer. However, the physical properties of both layers can improve this adherence. More integrated polymeric bilayer is achieved in the case 877 of choosing polymers with high mechanical strength for inner layer and polymer with high 
stretching property for outer layer. This combination stretches the outer layer on the tough support surface $[71,112,113]$.

880

The porosity of both layers is a crucial point for fabrication of a high flux Janus membrane.

Due to the fact that most of polymeric layer are fabricated through phase inversion process, the coagulation bath condition is the most important parameter to adjusting the porosity of the membrane. In this state, the composition of coagulation bath, its distance from the spinneret, and also its temperature should be properly adjusted in an optimum value. Coagulation bath containing a mixture of nonsolvent (water) and polymer solvent reduces the rate of phase inversion and directs the transition phase towards more porous structure. However, very slow phase inversion causes more dense structure in inner sides of the polymer layer and achieves opposite result.

Bonyadi et al. fabricated a Janus membrane by co-extrusion of PVDF and PAN solution in the outer and inner orifice layers of hollow fiber spinneret. The membrane showed no obvious defects in the layering and morphology. Due to the difference in expansion coefficient and also loosing of interactions after swelling of both layers, they were delaminated during the DCMD test and separation process was interrupted. In another attempt to modify this drawback, they fabricated a bilayer Janus hollow fiber membrane by using PVDF as main polymer and hydrophobic and hydrophilic clays in both dope solutions. In this study, PVDF solution containing hydrophobic cloisite nanoparticles have been used as outer layer and PVDF-PAN dope solution containing hydrophilic cloisite NA as the inner solution. The presence of PVDF in both layers caused strong adhesion through co-extruding process. In this study the effect of coagulant bath composition was studied and results demonstrated that a dense and smooth surface is obtained by using a nonsolvent with strong exchange rate, like 
901

902

903

904

water. But using a moderate coagulant bath like mixture of methanol and water made a rough membrane with more porous membrane structure. The optimum membrane porosity was achieved for coagulant bath comprising of water/methanol with concentration of $20 / 80$ wt\%, having contact angle of $140^{\circ}$ and $50^{\circ}$ for two sides [16].

\subsection{Electrospun Janus nanofiber membrane}

Electrospinning is a nanofiber fabrication method that uses electrostatic forces to produce ultrafine nanofibers, with high tolerance in tunability of the structure of produced mat [114, 115]. Also, the process can be easily controlled and different polymeric solutions or compositions can be used for the process. Having these features and also advantages of fast laboratory fabrication that increases the rate of optimising of the effective parameters made electrospinning and electrospraying as attractive methods for the fabrication of membranes. A bilayer electrospun Janus membrane can be fabricated by consecutive electrospinning of substrate and top layer. In this type of membrane, the special structure and morphology of electrospun membrane make good entanglement of electrospun nanofiber mats and produce a tough and strong bilayer composite [116]. It should be noticed that the composition of both substrate and top layer should be accurately chosen to find highest available interaction during layering and decreasing the possibility of delamination [117].

Some studies were performed using this method and usually a similar type of polymer for both layers is used. In other words, prepared composite dope solution for both layers have at least one similar polymer in its composition. Yue et al. fabricated a bilayer electrospun nanofiber firstly by electrospinning of PVDF-PVAc as hydrophilic substrate and then coated with electrospun PVDF nanofibers containing $\mathrm{SiO}_{2}$ nanoparticles modified by hexamethyl disilazane, as hydrophobic layer. In the prepared membrane, the presence of modified $\mathrm{SiO}_{2}$ 
enhanced the hydrophobicity of the coated layer, but decreased the interaction of ENFs with

the substrate layer. Therefore, in order to increase the layer interaction, it is possible to stepwise increase the concentration of modified $\mathrm{SiO}_{2}$. At first dope solution having lower nanoparticle percentage makes stronger interaction with substrate and then dope solution having higher percentage of nanoparticles is electrospun, which increases the hydrophobicity of outer surface. Membrane analysis showed WCA increment to $170^{\circ}$ and sliding angle decrement to $3^{\circ}$ by increase in nanoparticle concentration to $2 \mathrm{wt} \%$ [117]. The advantage of electrospun nanofibers is its intrinsic surface roughness that formed due to the cylindrical shape of nanofibers and multilevel structures of mats. This structure naturally increases the resistivity of the membrane against wetting problem by decreasing the interaction area and transferring the wettability state of the membrane toward the Cassie-Baxter state. Furthermore, coating of the membrane surface by nanoparticles can enhance its roughness makes electrospun nanofibers a good candidate for MD process [118].

Electrospinning is a novel fiber fabrication technique that has the advantage of great aspect ratio. The nanoscale diameter of the nanofibers increases the processibility of the fibers for various applications. This feature makes possible to adjust the pore size of produced mat for special applications. Due to micron-size pores of fabricated mats, electrospun nanofibers can be used in MD application. Also, the layer by layer fabrication process increases the roughness of produced mats, which is highly favourable for fouling and wetting resistivity of the membranes in MD operation. This configuration can be effectively applied in fabrication of

945 Janus membrane. The electrospinning process gives the option to coat hydrophilic electrospun nanofiber on top of superhydrophobic substrate. Also, by changing the applied 947 voltage and physical condition of solution, it is possible to electrospray the solution on top of 
the membrane substrate. Electrospraying can also produce a uniform, stable, and tough covering layer and it can be used for the implantation of miscrosphere structures on the surface of membranes to increase their roughness to produce omniphobic or superhydrophobic membranes. Electrospinning or electrospraying can also cover a uniform and even coating on the membrane surface and decrease the presence of defects on the membrane surface. These defects are places for intrusion of oil pollutants or surfactants that decrease the efficiency of the membrane for long term operation. Regarding these privileges, electrospinning and electrospraying have been widely used for fabrication of Janus membrane for water treatment application [103, 119-121].

Zhu et al. used both electrospinning and electrospraying methods for the fabrication of breathable asymmetric Janus membrane for MD application [33]. In his study, PVDF nanofibers was first spun as membrane substrate and then Si and low surface energy agent (FAS) were added to the solution containing PVDF and polystyrene and electrosprayed to transfer the omniphobicity to the membrane surface. Afterwards, $\mathrm{SiO}_{2}-\mathrm{PAN}$ solution was electrosprayed on the omniphobic substrate to fabricate Janus membrane. Proper selection of solution mixtures is an important aspect of this method. The microspheres coated for increment of roughness and decreasing of surface energy in first electrospraying step and coating of hydrophilic layer in second electrospraying stage should have adequate adhesion and durability. The attachment of polymer on top of solid polymeric layer is not very strong and it needs to consider the process of adhesion of coating layer on membrane substrate. The type of solvent used on the dope solution and its interaction with substrate polymer are important factors for increasing the stability of the coated layer. The solvent should have the ability to make a medium for interaction of quest polymer with substrate polymer.

971 Furthermore, as much as the produced microspheres are smaller, the stability, robustness, 
and roughness of the coating layer is higher. Therefore, when electrospraying technique is used for membrane fabrication, the process is affected by polymeric dope composition, applied voltage, tip-substrate distance, and other operational parameters that should be adequately adjusted to produce smaller size microstructures. Studies revealed that the addition of nano or micro size particles can break-up the production of larger-size microspheres during electrospraying process [122]. In an example, aerogel was added to the PVDF dope solution for electrospraying process. The hydrogel particles in high voltage electrospraying condition can disrupt the PVDF solution and distribute through the solution and make nano-sized spheres sprayed on the surface of substrate. Lower weight and higher contact area of sprayed microspheres helped increasing the interaction with substrate and enhanced durability and roughness of produced membranes.

Therefore, some additives or a mixture of some polymers should be used to simultaneously attain the desired property and strong adhesion and long durability. For example, LiCl was added to the PVDF solution of substrate to increase its conductivity that ease facile fabrication of electrospun nanofibers. Also, polystyrene have been added to PVDF-HFP-Si NPs to produce fibers having stabilized microsphere shape beads [122]. In a study, Wu et al used subsequent electrospinning method for fabrication of Janus membrane by deposition of hydrophilic PVA on PU substrate. PVA has the hydrophilic nature and PU has hydrophobic and their combination resulted to a high performing Janus membrane with $120^{\circ}$ contact angle difference between two sides. The processibility of electrospinning can also give the option to analyse the performance of Janus membrane having different coating thickness, by changing deposition time $[123,124]$. 


\subsection{Hydrophilic-Hydrophobic/Omniphobic Janus membrane configuration}

997

998

999

1000

1001

1002

1003

1004

1005

1006

1007

1008

1009

1010

1011

1012

1013

1014

1015

1016

1017

In a study carried out by Li et al., neat hydrophobic and Janus membranes were fabricated and their performance was compared. While Janus membranes with hydrophobic PVDF substrate showed highest flux, its salt rejection was lower than Janus membranes with omniphobic substrate. The covering of the PVDF substrate with fluorinated layer enhanced the salt rejection of the Janus membrane. Also, the comparison of the flux of Janus membranes with hydrophobic and omniphobic membrane showed that the Janus membrane has higher separation performance for both flux and salt rejection [53]. Experimental results showed that while the flux of hydrophobic membrane was $15 \mathrm{LMH}$ and it decreased to 14 LMH for omniphobic membrane, the Janus membrane showed flux of about $20 \mathrm{LMH}$ without compromising the salt rejection. These characteristics can be attributed to the presence of hydrophilic layer, which helps bring the hot feed water into the pores of the omniphobic membrane substrate for evaporation. Furthermore, in the case of using low concentration of hydrophilic solution, these materials can intrude into the beneath pores without blocking of the pores and decrease the thickness of hydrophobic or omniphobic layer and therefore increase the mass transfer ratio. On the other side, higher concentration of the coating layer can block the pores and decrease the flux $[32,69]$. The blockage of the pores by top layer should be recognised on the reasons for flux decrement. For example, in one study, the flux of the fabricated membrane decreased from $29 \mathrm{LMH}$ to $17.5 \mathrm{LMH}$ by covering of the hydrophobic membrane by a hydrophilic layer. Though the permeate conductivity of the Janus membrane remained constant during the $600 \mathrm{~min}$ test, the hydrophobic membrane lost its salt rejection performance and permeate conductivity reached from zero to $200 \mu \mathrm{S} / \mathrm{cm}$ 
[27]. However, the comparison of different experimental studies have demonstrated that the value of flux decreasing is dependent on the coating procedure and also the type of used materials and in some cases the flux of Janus membrane have enhanced $[30,33,53]$. Zhu et al. have shown that while the flux of the Janus membrane is relatively equal to the hydrophobic ENF PVDF (28 LMH), its salt rejection is stable for long term application and permeate conductivity remained approximately zero during $50 \mathrm{hrs}$ test experiment. This value for hydrophobic ENF PVDF reached to $75 \mu \mathrm{S} / \mathrm{cm}$ after $50 \mathrm{hrs}$ [33].

Generally, the explanation of any process for fabrication of Janus membrane should be assessed case by case. Although the presence of thin hydrophilic layer can improve the thermal efficiency of the MD module, increment of the hydrophilic layer thickness above an optimum value can have opposite influence. In this case, temperature polarization worsens and thick solid layer decreases the rate of water passage and, as a result, the flux decreases. Furthermore, the heat from the feed side cannot easily transfer to the boundary layer of evaporation and this barrier decreases the driving force of vapour transport. In general, the degree of hydrophilicity of the top layer is an important factor for determination of the influence of the Janus membrane in separation of wastewater. Hydrophilic layer of Janus membranes with lower hydrophilic affinity has less feed transport rate and consequently the amount of water reached to the surface of hydrophobic layer for vaporization decreases and the flux of the membrane decreases. Therefore, the selection of top layer with high wettability is desired. Also, the saturation time for the top layer is an important factor during lab work and the experimental data should be reported after saturation of top layer $[50,60]$. In this way, during recent years, various materials have been used in different studies and a broad experimental data of the performance of fabricated Janus membranes was obtained. A general comparison of different studies performed by different coating materials showed 

relatively higher effectiveness of zwitterionic hydrophilic layer for decreasing of fouling 1043 problem and increasing separation performance. In other words, the zwitterionic made 1044 strong adhesion on omniphobic membrane without compromising the omniphobicity of 1045 substrate layer [27]. A brief comparison of different Janus membrane fabricated for MD 1046 application is shown in Table 1. 
Table 1. Comparison of different membrane optimization methods in terms of MD permeate flux.

\begin{tabular}{|c|c|c|c|c|c|c|c|c|c|c|c|}
\hline $\begin{array}{c}\text { Hydrophilic } \\
\text { layer }\end{array}$ & 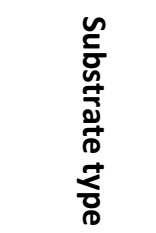 & $\begin{array}{l}\text { Hydrophobic } \\
\text { Layer }\end{array}$ & Method & $\Delta T$ & $\begin{array}{l}\frac{\pi}{\bar{x}} \\
\frac{x}{x} \\
\frac{5}{x}\end{array}$ & Advantages & $\begin{array}{l}\text { Dis- } \\
\text { advantages }\end{array}$ & Module & $\begin{array}{l}\frac{1}{\frac{1}{2}} \\
\frac{0}{0} \\
\frac{0}{0} \\
\frac{0}{0} \\
\frac{0}{n}\end{array}$ & 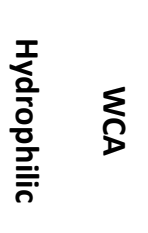 & Ref \\
\hline $\begin{array}{c}\text { CTAB/PVDF- } \\
\text { HFP }\end{array}$ & $\begin{array}{l}\text { Nano } \\
\text { Flber }\end{array}$ & $\begin{array}{c}\text { SiNP/CTS/PFO } \\
\text { Spray }\end{array}$ & $\begin{array}{l}\text { ENF/Dip } \\
\text { coating }\end{array}$ & 50 & & & & DCMD & 150 & 10 & [48] \\
\hline $\begin{array}{l}\text { Dopamine -self } \\
\text { polymerization } \\
\text { (L) }\end{array}$ & $\begin{array}{l}\text { FLAT } \\
\text { SHEET }\end{array}$ & PTFE/PP (B) & Dip coating & 50 & 19.7 & $\begin{array}{l}\text { Long term } \\
\text { performance } \\
\text { Low fouling } \\
\text { and wetting }\end{array}$ & $\begin{array}{c}\text { Fabrication } \\
\text { difficulty } \\
\text { (3 step) }\end{array}$ & DCMD & 128 & 30 & [64] \\
\hline Zwitterionic & $\begin{array}{l}\text { HOLLOW } \\
\text { FIBER }\end{array}$ & $\begin{array}{l}\text { Omniphobiced } \\
\text { Quartz fiber }\end{array}$ & & 40 & 15.5 & $\begin{array}{c}\text { High } \\
\text { antifouling } \\
\text { and } \\
\text { antiwetting }\end{array}$ & $\begin{array}{c}\text { Fabrication } \\
\text { difficulty } \\
\text { (3 step) }\end{array}$ & DCMD & 121 & 25 & [27] \\
\hline $\mathrm{SiO}_{2} / \mathrm{PAN}$ & $\begin{array}{l}\text { Nano } \\
\text { Flber }\end{array}$ & PVDF ENF & $\begin{array}{l}\text { Elstrospinning } \\
\text { Electrospraying }\end{array}$ & 40 & 25 & $\begin{array}{c}\text { excellent } \\
\text { breathability }\end{array}$ & $\begin{array}{c}\text { Low } \\
\text { mechanical }\end{array}$ & DCMD & 170 & 10 & [33] \\
\hline
\end{tabular}




\begin{tabular}{|c|c|c|c|c|c|c|c|c|c|c|c|}
\hline & & $\begin{array}{l}\text { (F:SiO2/PAN, } \\
\text { M: SiNP FAS } \\
\text { PVDF PS, P: } \\
\text { PVDF-HFP) }\end{array}$ & & & & $\begin{array}{c}\text { high } \\
\text { antifouling }\end{array}$ & & & & & \\
\hline $\mathrm{PDA}^{-\mathrm{AgNO}_{3}}$ & $\begin{array}{c}\text { HOLLOW } \\
\text { FIBER }\end{array}$ & PDA-AgNPs & HF-Coating & 40 & 17 & $\begin{array}{l}\text { Antibacterial } \\
\text { Long term } \\
\text { operation }\end{array}$ & $\begin{array}{l}\text { Presence of } \\
\text { Ag in } \\
\text { permete }\end{array}$ & DCMD & 109 & 7.6 & {$[82]$} \\
\hline PVA-CNT & $\begin{array}{c}\text { FLAT } \\
\text { SHEET }\end{array}$ & PVDF & spraying & 40 & & $\begin{array}{l}\text { High fouling } \\
\text { resistence }\end{array}$ & CNT stability & DCMD & 115 & 33 & [34] \\
\hline PDA-PEI & $\begin{array}{l}\text { HOLLOW } \\
\text { FIBER }\end{array}$ & PVDF & Dip Coating & 40 & 15 & $\begin{array}{l}\text { one-step } \\
\text { fabrication }\end{array}$ & $\begin{array}{c}\text { pore } \\
\text { blockage }\end{array}$ & DCMD & 109 & 25 & [57] \\
\hline $\mathrm{PI}$ & $\begin{array}{l}\text { FLAT } \\
\text { SHEET }\end{array}$ & PTFE & Dip Coating & 40 & 8 & $\begin{array}{c}\text { low cost, one } \\
\text { step, }\end{array}$ & Low Flux & DCMD & 145 & 45 & [98] \\
\hline $\begin{array}{c}\mathrm{KOH}- \\
\text { Modification }\end{array}$ & $\begin{array}{l}\text { FLAT } \\
\text { SHEET }\end{array}$ & PVDF & grafting & 50 & 45 & $\begin{array}{l}\text { Simple } \\
\text { Process }\end{array}$ & low durability & DCMD & 150 & 62 & {$[61]$} \\
\hline
\end{tabular}




\begin{tabular}{|c|c|c|c|c|c|c|c|c|c|c|c|}
\hline Cotton Fabric & $\begin{array}{l}\text { FLAT } \\
\text { SHEET }\end{array}$ & PDMS & grafting & & & $\begin{array}{l}\text { high } \\
\text { wettability } \\
\text { difference, }\end{array}$ & $\begin{array}{c}\text { complicated } \\
\text { process }\end{array}$ & & 153 & & [87] \\
\hline $\begin{array}{l}\text { PVDF-PAN- } \\
\text { Cloisite }\end{array}$ & $\begin{array}{c}\text { HOLLOW } \\
\text { FIBER }\end{array}$ & PVDF-cloisite & CO-extrusion & 90 & 55 & High Flux & & DCMD & 50 & 140 & [16] \\
\hline Hydrogel & $\begin{array}{l}\text { FLAT } \\
\text { SHEET }\end{array}$ & Teflon (PTFE) & Coating & 40 & 30 & $\begin{array}{l}\text { High wetting } \\
\text { resistivity }\end{array}$ & Low stability & DCMD & & & [55] \\
\hline PDADMAC/PAA & $\begin{array}{l}\text { FLAT } \\
\text { SHEET }\end{array}$ & PVDF & & 45 & 5 & $\begin{array}{l}\text { long-term } \\
\text { robustness }\end{array}$ & Low flux & DCMD & 125 & 50 & [63] \\
\hline DA & $\begin{array}{l}\text { FLAT } \\
\text { SHEET }\end{array}$ & PTFE & & 50 & 89 & $\begin{array}{l}\text { High flux, } \\
\text { Antifouling } \\
\text { resistivity }\end{array}$ & & VMD & 134 & 59 & [125] \\
\hline $\begin{array}{l}\text { cellulose } \\
\text { acetate }\end{array}$ & $\begin{array}{l}\text { FLAT } \\
\text { SHEET }\end{array}$ & PTFE & Electrospinning & 40 & 20 & $\begin{array}{l}\text { Antifouling, } \\
\text { Mechanical } \\
\text { strength }\end{array}$ & & DCMD & 135 & 39 & [126] \\
\hline graphene oxide & $\begin{array}{l}\text { FLAT } \\
\text { SHEET }\end{array}$ & PVDF & dip-coating & 40 & 25 & $\begin{array}{l}\text { Antifouling } \\
\text { and } \\
\text { antiwetting }\end{array}$ & Low stability & DCMD & 145 & & [127] \\
\hline
\end{tabular}




\begin{tabular}{|c|c|c|c|c|c|c|c|c|c|c|c|}
\hline PU & $\begin{array}{l}\text { FLAT } \\
\text { SHEET }\end{array}$ & PTFE & Dip coating & 40 & 23 & $\begin{array}{l}\text { Antifouling } \\
\text { and } \\
\text { antiwetting }\end{array}$ & & DCMD & 130 & 15 & [128] \\
\hline EDA/PEI & $\begin{array}{l}\text { FLAT } \\
\text { SHEET }\end{array}$ & PVDF & $\begin{array}{l}\text { Electrospinning } \\
\text { and dip- } \\
\text { coating }\end{array}$ & 35 & 5 & $\begin{array}{l}\text { High oil- } \\
\text { fouling } \\
\text { resistivity }\end{array}$ & Low flux & & 127 & 40 & [78] \\
\hline
\end{tabular}


1110

1111

1112

Always, nature is the best reference for the production of most optimum and environmental friendly materials and instruments. Mussels have the ability to make a strong, rapid and tough adhesion to different underwater places and preserve themselves from high pressure water forces. This tough adhesive that has the ability to adhere to various surfaces has dopaminebased structure and as a result, mussels as inspired from nature have been utilized for the fabrication of materials to render attachment of different surfaces having opposite wettability tendency. In fact, dopamine has the ability to be used as an interface for attachment of hydrophilic layer on top of hydrophobic membrane substrates $[30,107]$. Polydopamine is a biological adhesive inspired from mussels and formed through self-polymerization of dopamine at room condition via autoxidation in an aqueous media containing dissolved oxygen [59, 129]. The presence of catechol, quinone and amine functional groups gives hydrophilic nature to dopamine and also make it possible to make various types of interaction, including hydrogen bond, covalent bond, pi-interaction, charge transfer interaction and metal chelation, with substrate materials. This wide range of interaction made dopamine an attractive material for adhesion to substrates having various condition like wet, dry, organic, or inorganic. This ability caused PDA play the role of main or intermediate for covering of top layer of hydrophobic, superhydrophobic, or omniphobic membranes for fabrication of Janus membranes in MD applications [12, 59, 129]. For example, Chew et al. have co-deposited a hydrophilic layer of PDA/PEI on the outer surface of PVDF hydrophobic substrate. The novelty in this work is in the co-deposition of hydrophilic layer on top of hydrophobic substrate. The hydrophilic layer made strong bond with water molecules and become hydrated and this hydrated layer prevent membrane fouling and enhance the separation performance of the membrane [57]. In this work, the permeate flux for Janus membrane (11 LMH) was slightly lesser than hydrophobic ones (4 LMH), but the permeate 
conductivity for Janus membrane was close to zero during $80 \mathrm{hr}$ test ( $100 \%$ rejection) and for hydrophobic membrane it stepwise increased until it reached to more than $1000 \mu \mathrm{S} / \mathrm{cm}$ after $20 \mathrm{hrs}$

In another study, Wu et al also deposited PDA/PEI on the surface of PP membrane. In this work, Janus membrane was fabricated by floating of a PP membrane on solution containing dopamine and PEI. As a result, the hydrophilic layer was deposited on the substrate using mussel-inspired catechol groups of dopamine. This asymmetric configuration obtained $130^{\circ}$ wettability difference between two sides of the membrane [59]. The SEM images proved the presence of microchannel which helped the transport of water molecules using hydrophilic moieties and capillary forces. Also, the MD experimental results showed that while the pore size of the outer surface of the Janus membrane compared to neat hydrophobic membrane decreased, the flux did not decrease. This result proved that the proper selection of type and method of top hydrophilic layer cover its drawbacks. In addition, long-term experimental results in high SDS feed water better determined the effectiveness of the prepared Janus membrane. While the neat hydrophobic PVDF membrane encountered severe wetting and fouling by increment in permeate conductivity and decrement in flux after $90 \mathrm{~h}$ of test, the Janus membrane continued its good flux and salt rejection. This result demonstrated that the grafted hydrophilic layer can positively prevent the importation of surfactant and oil droplets and can prevent the Janus membrane from fouling and wetting [59].

Furthermore, in another study, a one-step fabrication of Janus membrane was tried by using SMM. In a series of experiments, SMMs were fluorinated and then mixed with polyetherimide to fabricate Janus membranes. The results showed that the derived composite membrane reached LEP greater than 2.9 bar. However, both liquid and gas flux of the membrane 
decreased due to lower pore sizes of modified membrane. In addition, the DCMD results of

1158 Janus membranes prepared by PEI and SMM in different studies showed equivalent or higher

1159 flux compared to commercial PTFE membrane $[17,96]$

1160 The treatment of wastewaters with high microorganisms content is always challenging. For this type of wastewater, it is applicable to use some nanoparticles that have antibacterial activity to simultaneously improve separation performance of MD membrane and decrease bacterial growth on the surface of the membrane. In an experiment, hydrophilic PDA layer was coated on top of hydrophobic PVDF hollow fiber to fabricate a Janus membrane. Afterwards, to increase both antibacterial activities and fouling resistivity of the membrane, silver nanoparticles were used as top layer coating. The experimental study on the prepared membrane proved the lower adhesion of fouling agents on the membrane surface. In addition, the proliferation of sulphur-containing proteins or thiol groups of enzymes was restrained in the silver-containing Janus membrane, which proves its antibacterial activities [82].

\subsection{Hydrophobic-hydrophilic Janus membrane configuration}

Though most of research on Janus membrane were performed to investigate the effect of hydrophilic layer on feed side of the MD system, some studies have investigated the impact of hydrophilic layer on permeate side of the MD modules. In these works, the hydrophilic face of the Janus membrane was placed toward permeate side. In a study performed by Zou et al, a hydrophilic layer containing PEG was coated on hydrophobic substrate and separation and energy performance of prepared Janus membrane was investigated by placing its hydrophilic side toward permeate side of the module, while the feed side of the membrane was 
hydrophobic. The change in energy performance of the Janus membrane was compared to neat hydrophobic membrane and results proved that co-extruding of a thin PVDF/PEG hydrophilic layer on the PVDF hollow fiber substrate enhanced the energy efficiency of the membrane from $55 \%$ to $72 \%$. This increase can be attributed to the presence of highly porous and hydrophilic layer on permeate side that improves the condensation rate of evaporated layer. Also, the hydrophilic layer prevents the intrusion of permeate water into the pores and decrease the wetting possibility. Experimental results revealed that the salt rejection, flux and heat efficiency of the membrane have decreased in long term test and wetting problem occurred after $200 \mathrm{~h}$ of operation [71]. In general, in this type of Janus membranes, the bottom layer should have high heat conductivity to easily transfer the released heat of condensation and maintain the driving force of water transport. In this type of configuration, the hydrophilic layer plays the role of increasing the mass transfer, decreasing the heat loss, and increasing the mechanical strength of the membrane $[60,89,130]$.

\section{Challenges facing Janus membranes in MD}

Regardless of the type of method used for fabrication, Janus membranes have encountered two main challenges, which make problems in the way of its performance and potential commercialization. First, due to different chemical structure of hydrophobic and hydrophilic layers, Janus layers have weak interaction with each other and have been delaminated after a period of time of operation. This poor compatibility is a crucial point for long term application of Janus membranes. Second, only the hydrophilic layer can repel hydrophobic foulant and low surface compounds, like surfactants, and if these foulant can pass through 
the hydrophilic layer, they can cause wetting of the hydrophobic layer beneath $[22,30,33$, 71].

\subsection{Delamination}

1205

Delamination is one of the main issues facing the use of Janus membranes. As per design structure of a Janus membrane, it consists of two layers with opposing wettability, which means that the layers could be made of different materials that are not affinitive with each other without proper modification. For example, in general Janus membrane fabrication, the hydrophobic substrate is firstly modified to form re-entrant structure on the surface and coated with low surface energy materials converting it into omniphobic membrane. The omniphobic membrane then becomes the substrate to coat a hydrophilic layer on top to form the Janus membrane, but the low surface energy coating on the omniphobic membranes

1213 decreases the interaction of coated hydrophilic layer and a weak adhesion is formed that make it less robust and stable. This problem is exacerbated during the water treatment

1215 process when the top layer becomes hydrated. Due to difference in wettability of the contacted layer, the swelling of hydrophilic layer increases interaction conflicts and may cause membrane delamination problems. Therefore, high attention should be paid to simultaneously improve layer adhesion and separation performance of Janus membrane [32, 37, 38]. The schematic of delamination process is shown in Figure 10. 




1222 Although the fabrication of multi-layer polymers has been widely progressed in recent years, 1223 delamination still remained as an issue in this process. In general, the difference in physical and chemical properties of both layers make it a balancing challenge between attachment and detachment forces. Also in some studies, in order to increase the accuracy and quality of the top layer, the hydrophilic layer was coated using layer-by-layer method and through some steps. This layering coating causes distribution of exerted stress across the coated layer and, in real operational condition, sequential detachment takes place for a multilayer surface (as shown in Figure 10). The delamination in coating process generally refers back to one of 
following two mechanisms: (1) difference in phase inversion process during coagulation step, and (2) difference in shrinkage coefficient of layers in both sides of coating layer. In order to decrease the delamination, the best choice is by selecting dope solution coming from similar solvents and/or the same polymer family. Using similar solvent helps adhesion of distinct layers to form an integrated coating. For example, in a study, $\varepsilon$-Caprolactam was used as solvent for both PVDF hydrophobic substrate layer and PVDF-PVA hydrophilic top layer. The results demonstrated that using water-soluble solvent improved the fabrication of delamination-free Janus membrane for MD application. The presence of same solvent and PVDF polymer in both hydrophobic and hydrophilic layer and also the solubility of $\varepsilon$ Caprolactam in the water resulted in the fabrication of an integrated Janus membrane [67]. However, MD test results for some experiments indicated that this method compromised the wettability of coated or substrate layers. As a result, the separation performance is lower compare to Janus membranes having high wettability difference between two layers.

Another issue during phase inversion process of Janus membranes is that the structure and phases of layers can change. If the two layers are formed with different coagulation rates, two separate layers are formed that have low attachment with each other. Therefore, besides the selection of dope compositions and their compatibility, the ternary phase inversion of both layers should be compared to find the difference in phase inversion rates. Furthermore, Janus membranes operated in temperatures below or beyond the ambient temperature has led to shrinkage or expansion during the process. This indicates that one has to consider the differences in shrinkage coefficient that can amplify the stress and strains on the polymers and on the coated layer that further exacerbate layering and delamination problem $[41,131]$. To address this drawback, many groups have used various new fabrication techniques, like co-extrusion for hollow fiber membranes, co-casting for flat sheet membranes, addition of 
diluents or additives, and proper selection of polymers according to their phase diagram [14, $132-134]$

In general, the quality and robustness of coated layers to avoid delamination problem are tested via exposing them to harsh conditions. One way is by immersing the membrane in an ultrasonic bath for a long time, which exposes it to constant stress and strain from ultrasonic waves. Analysis is then carried out to determine the condition and amount of the coated layer with respect to its previous condition. One study utilized FTIR to determine the functional groups on a coated PVDF Janus membrane after exposing to ultrasonicaiton for 10 minutes. The results showed the presence of functional groups that make strong attachment between layers, which were corroborated by the EDX and DCMD results $[32,82]$.

To enable good interaction of the hydrophilic layer with the substrate for strong adhesion, some groups used a pre-treatment process to prepare an omniphobic substrate for strong coating step [59, 82]. Studies have shown that a hydrophilic dope solution needs active sites on the omniphobic layer to attach to it. Direct coating of top hydrophilic layer will not render it effective due to the absence of active sites on the previously generated omniphobic surface. This is because omniphobic substrates naturally repels almost all types of liquid and do not let the solvents to make active sites. Forming fluorocarbon sites on the omniphobic surface is a good strategy but it needs high activation energy, equivalent to a temperature of $90^{\circ} \mathrm{C}$. The

1272 plasma etching technique is a good pre-treatment method to effectively produce active sites on the omniphobic surface prior to coating of hydrophilic layer. A study has utilized such pretreatment strategy and strong interaction between the coating layer and the omniphobic layer was observed, and no delamination was found after DCMD test. Compared to other 
fabricating techniques of Janus membranes, high and stable MD separation performance was observed for this Janus membrane [74].

\subsection{Reduced vapour transport}

Another challenge for Janus membranes, which usually comes along with delamination problem, is dense interface morphology issue that provides resistance against vapour transport across the membrane for MD application. The compactness at the interface of coating layer generally decreases the effective surface area, and as a result decreases the flux of the membrane. For example, Lin et al. coated a porous hydrophobic PTFE membrane by hydrophilic hydrogel to enhance its antifouling and anti-wetting performance, but the compactness and blockage of the pores decreased the flux of the membrane for DCMD application from $30 \mathrm{LMH}$ to $23 \mathrm{LMH}$ [55]. In another study, Wang et al utilized chitosan to modify hydrophilic PVDF membrane and results showed $15 \%$ flux decline with respect to neat PVDF membrane (reached to 26 LMH from 31 LMH) [52]. However, the modified membranes showed significantly better antifouling and antiwetting performance. One potential way to address both delamination and dense interface morphology problems is by manipulation of the concentration and composition of dope solution and also fabrication using co-extrusion method. Zuo et al. have used this method and fabricated a membrane with different dope compositions to find the most optimum point for decreasing the delamination [111]. The dense interface morphology was overcome by addition of alumina nanoparticles to the inner layer of co-extruded orifice. In this condition, alumina nanoparticles made some defects on the polymeric matrix layer and increased the porosity and, consequently, decreased the dense morphology. This resulted to enhanced flux performance. However, overuse of nanoparticles also decreased the mechanical strength, the separation performance, and the 
top-layer attachment to the membrane [111]. Thus, balance must be performed when designing a Janus membrane material with regards to the structure of the coating layer while maintaining strong adhesion to the substrate material.

\subsection{Scaling problem in Janus membrane}

In the DCMD process, during water vaporization and vapour transport across the pores, the salt concentration in the membrane-feed water interface increases. Additionally, due to heat loss through the membrane matrix and also latent heat conversion, the temperature of feed layer close to the surface decreases and is lower than the temperature of the bulk. This situation transfers concentrated water into supersaturated zone and prepares condition for formation of mineral scaling by deposition of excess minerals at the interface layer. Therefore, due to intrinsic hydrophilicity of the formed scales, the hydrophobicity of the membrane surface decreases, that can increase the probability of wetting and diminish LEP of the membrane. Also, further increase in scales can block the membrane pores and simultaneously decrease the flux of the membrane. Therefore, scale formation, which has separate characteristic from fouling problem, should be observed for long-term operation of the MD membranes $[71,135]$. One of the commonly used method for cleaning the scaling on the surface of the membranes is membrane regeneration. In this method, after a period of time, the scales are physically or chemically removed from the membrane surface. Although the membrane regeneration increases the operability of the membrane, but in general, due to potential change in physical and chemical structure of the membrane pores, the performance of the regenerated membrane is not similar to the neat membrane. All in all, the most cost effective method is preventing of formation of scales on the membrane surface [71]. 
Due to high salinity and contamination of feed water in MD process, scaling is highly susceptible to form and is one of the major problems of MD modules. According to nature of feed water, different types of scales may be formed. The most common types of MD scales are calcite, gypsum, and silica. Gypsum and $\mathrm{NaCl}$ scales form through crystallization mechanism, while silica scale is formed by polymerization of silica acid, a non-crystallization method. Anti-scalants are widely used to mitigate the crystallization and formation of scales, but due to amorphous structure of silica minerals, most of used anti-scalants showed weak performance for decreasing silica scale formation $[60,136]$. Presence of silica scales have wastewaters $[137,138]$.

In general, the scaling can be formed through two pathways: homogenous or heterogeneous nucleation. In homogenous nucleation scaling, by supersaturating of minerals in small liquid parts, the scale particles re spontaneously formed on that place. By increasing the concentration of minerals by evaporation of liquids, the homogenous scaling continues to form and formed scales deposit on the surface of membranes. Heterogeneous scaling is another type of scales that are formed at the liquid-solid interface. In this type of scaling, the interaction between dissolved minerals and membrane surface play the main role for scale formation. Therefore, the physical and chemical characteristics of the membrane surface determine the level of scaling $[58,139]$.

Until now, different studies have focused on mitigation of fouling and wetting in MD process, usually by organising roughness of the surfaces and coating with low surface energy materials. Though these modification methods could effectively diminish fouling and wetting problems, but the scaling challenges still need additional attention for commercialization of MD 
membranes $[105,139]$. In order for deep investigation of the scaling issue, the composition of fresh and used membranes should be analysed by EDX to investigate the type of crystals formed on the surface of the membrane after wastewater treatment by MD. In a study performed by Zou et al., the new peaks were observed in the used membrane, which were relevant to the oxygen, irons, calcium, and magnesium. The results demonstrated that sulfate, carbonate, and hydroxide scales are formed on the membrane surface. Comparison of the intensity of the peaks revealed that the calcium carbonate is one of the main scales formed on the surface of membrane. However, the membrane used for treatment of RO brine usually have large proportion of sodium chloride scales and the composition of wastewater determine the most dominant scales formed on the membrane surface $[71,140]$.

Additionally, the silica formation is another major scaling problem in application of $M D$ process. Although the formation of homogenous nucleation depends on the characteristics of feed water, the main challenge of scaling is formation of heterogonous nucleation that attach to the membrane surface and gather homogenous silica scales and increase the volume of scales on the surface. For this reason, different studies were carried out to decrease the side effect of scale formation by enhancement of membrane surface geometry and structure to decrease slippery angle of the membrane, which directly cause decrease in formation and attachment of heterogeneous nucleation on the membrane surface $[141,142]$. In a study, Yin et al. performed a DCMD test to investigate the behaviour of Janus and hydrophobic membranes during scale formation in a high SDS feed water. The feed solution containing different concentration of amorphous silica, $\mathrm{NaCl}$, and gypsum scales was prepared and the test was performed on three different membranes: hydrophobic PVDF membrane, PVDF-SiNP-FAS superhydrophobic, and PVA/PVDF-SiNP-FAS Janus membrane. The sliding test was performed on the membranes and results show that while hydrophobic 
membrane showed high WCA, the water droplet did not slide from the membrane surface.

Furthermore, water droplet starts to slide at sliding angle of $17^{\circ}$ for superhydrophobic membrane [105].

Also, dynamic light scattering (DLS) test was used to determine the hydrodynamic diameters of the scales formed during the MD test. All membranes showed perfect separation performance during DCMD test for feed water with initial saturation index of -0.82 . In this condition, only small heterogeneous silica scales were formed on the membrane surface, without affecting the performances of the membranes and structures of the pores. For the feed water with silica saturation index of 0.55 , gel-like silica scales with the size of $100-200$ $\mathrm{nm}$ were formed in all membranes and the flux decreased for all of them, but Janus membrane experienced lower decrease in performance. In this condition, due to high concentration of silica, the homogenous nucleation of silica particles can react with silica acids and form a cross-linked structures which can attach to the membrane surfaces. Continuous formation and attachment of this particles can clog the membrane pores and also change the wettability of membrane surface. As a result of this silica-silica interaction and formation of both heterogeneous and homogenous silica nucleation, the flux of the membrane decreased and the conductivity of permeate increased. The results of this experiments showed that the formation of scales decreased the flux of Janus membrane, similar to hydrophobic and superhydrophobic ones, by clogging the membrane pores. Eventhough the scale formation changed the wettability of the hydrophobic or superhydrophobic membranes, it did not change the wettability of the Janus membranes $[61,105]$. All in all, the flux decline in superhydrophobic membrane was less than others, but the Janus membrane derived the highest water productivity. In order to remove the formed scales on the membrane surface, the Janus membrane can be regenerated using custom backwashing method. Zou et al 
regenerated the Janus membrane and then analysed the membranes for evaluation of its performance. The experimental results showed that after $16 \mathrm{~h}$ of test, the water recovery of the membrane became about half of the fresh membrane after three days of continuous test. This result proved that the formation of scales on the Janus membrane deformed the structure of the pores and the stability and the shape of the membrane changed and lost its high salt rejection and water recovery in long term operation [71].

\subsection{Formation of microdefects on hydrophilic layer}

Besides the delamination problem, the coating of a hydrophilic layer on top of an omniphobic or a hydrophobic layer can face another challenge: the formation of microdefects during formation or polymerization of hydrophilic layer, which can produce microchannels, resulting to a decrease in salt rejection of the membrane. To address this challenge, the coated hydrophilic layer using common methods is usually exposed to drying and hydration steps before their application in real wastewater treatment. Also, sometimes during the real test, the membrane may go to a recovery or maintenance mode and get dried. The changes in hydration (consecutive drying and wetting) can affect the structure of the hydrophilic layer and produce some defects that decrease the performance of the membrane. For covering this deficiency, in some studies production of multilevel structure was suggested. Also according to Wenzel theory, similar to hydrophobic surfaces that higher roughness increases hydrophobicity of the membrane, roughness increment in hydrophilic layer can increase the hydrophilicity of the top layer. For example, Chew et al. fabricated a Janus membrane by coating of self-polymerized PDA layer on top of PVDF porous hydrophobic substrate. The wetting and fouling tests on the prepared membrane was carried out using $500 \mathrm{mg} / \mathrm{L}$ Tween 20-stabilized petroleum-in-water emulsions and results showed better performance for Janus 
membrane with respect to neat PVDF membrane. The neat PVDF membrane encountered

1416 severe wetting and fouling after $20 \mathrm{~h}$ operation, but the Janus membranes showed slight

1417 wetting and fouling after $50 \mathrm{~h}$ of experimental test. The analysis was carried out to determine

1418 the source of decrease in the efficiency of Janus membrane. In this way, the SEM images

1419 showed presence of microvoids that work like channels to transmit fouling and wetting agents

1420 to the hydrophobic layer beneath hydrophilic top layer and, therefore, decrease in the

1421 performance of the membrane can be attributed to these voids.

1422 In order to heal these features, Janus membrane can be coated with nanoparticles to cover

1423 the formed microchannels or prevent the formation of these microvoids during hydration

1424 stresses. In a study, the surface of hydrophilic layer was coated with Ag nanoparticles using

1425 immersion techniques (Figure 11). The experimental DCMD results showed perfect

1426 performance even after $96 \mathrm{hr}$ test without compromising the flux or salt rejection. The $\mathrm{Ag}$

1427 nanoparticles could effectively cover the microchannels and prevent entrance of low surface

1428 energy compounds or oil droplets, even in high oil and surfactant contaminated feed water $1429 \quad[82]$. 




PVDF Membrane

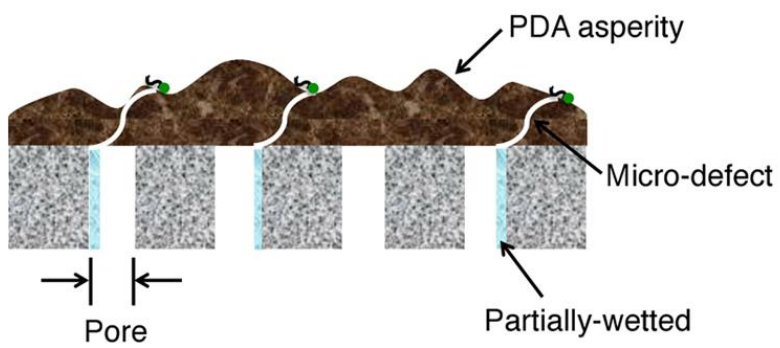

6h-S-mPVDF Membrane

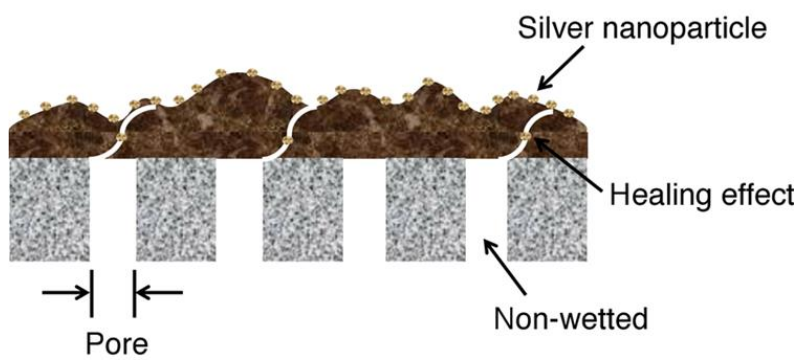

6h-M-mPVDF Membrane

1432

Figure 11. the effect of dispersion of silver NPs on the wetting resistance of Janus membrane

\section{Conclusions and future perspectives}

This review focused on the investigation of different aspects of fabrication, modification, and application of Janus membranes for membrane distillation (MD). Various methods for modification of the surface of the hydrophobic or omniphobic membranes were investigated and the benefits and challenges of methods were explored. Furthermore, different possible membrane configurations in various types of modules like flat sheet and hollow fiber have 
been introduced. The antiwetting and antifouling performance of the prepared Janus membranes in different types of feed water was investigated and the results were compared to neat hydrophobic membranes. The results showed higher efficiency of Janus membranes for treatment of feed waters having high concentration of contaminants and pollutants and proved potential of the Janus membrane for long-term application in MD systems.

However, Janus membrane still is dealt with some challenges that impede its overall performance and potential commercialization. First of all, Janus membranes is fabricated by modification of MD membranes that are microporous hydrophobic membranes, suitable for microfiltration process. These membranes still have some problems for commercialization and the first step is fabrication of a special type of membrane for MD. The second challenge refers to the types of materials used for modification of the membranes, which should be cheap, environmentally friendly, and easily processed. Furthermore, some preparation methods give good laboratory results but they do not have bright prospect for large scale production. Therefore, researches should be directed to prepare a more ecofriendly and producible Janus membrane. The mechanical stability of the Janus membrane is another important matter that needs to be recognised for enhancement of tolerability of the membrane for long-term working on harsh condition. Additionally, the mechanism of the transport of the liquid through the hydrophilic layer and phase change to vapour in Janus membrane have not been properly investigated and a detailed modelling or simulation is necessary.

Regarding attractive results derived from experimental research, the Janus membranes showed high potential to be a candidate for treatment of highly polluted, challenging wastewaters in MD systems. For this reason, the direction of the future researches should be 
accurately determined to cover the available deficiency of the membranes. The overview of the current researches on Janus membrane declared the void points in the research environment. The most important point for fabrication of defect-free Janus membrane is solving the delamination issues. Also, the change in configuration of the layers can be helpful to cover current deficiency of the Janus membranes. In this way the following suggestion are made as future prospects for the preparation and design of Janus membranes for MD:

- Using compatible materials that have good chemical integrity, but different hydrophobicity

- Fabrication of trilayer membrane that has hydrophilic affinity in both side but hydrophobic affinity in middle, which theoretically shows attractive configuration for enhancement of both heat and mass transfer efficiency

- Using dual-electrospinning technique that can bring asymmetric and high porosity membrane

- Using economic and non-fluorinated materials that has low environmental issues and low cost

- Focusing on one-step fabrication methods to ease fabrication process and decrease the processing costs like improvement of SMM based Janus membranes

- Most of studies were performed in flat sheet module and more research on other commercial modules like hollow fiber is necessary. 


\section{Acknowledgement}

1485 M.A. is thankful to the financial support for his PhD studies through the International 1486 Research Scholarship (IRS) and UTS President Scholarship (UTSP). L.T. appreciates the 1487 support from UTS CTWW internal fund. H.K.S expresses gratitude for the support by the 1488 Korea Environment Industry \& Technology Institute (KEITI) through Industrial Facilities \& 1489 Infrastructure Research Program, funded by Korea Ministry of Environment (MOE) 1490 (1485016424), and also from the Australian Research Council (ARC) support.

1492 List of abbreviations

1493 ALD

1494

1495

1496

1497

FAS

1498

1499

1500

MD

$1501 \quad$ NIPS

1502 NP

1503

OCA
Atomic Laser Deposition

Carbon Nanotubes

Dodecyl Trimethyl Ammonium Bromide

Electrospun Nanofiber Membrane

(Heptadecafluoro-Tetradecyl) Trimethoxysilane

HLB

Hydrophilic-Lipophilic Balance

Liquid Entrance Pressure

Membrane Distillation

Nonsolvent Induced Phase Inversion

Nanoparticle

Oil contact angle 
1504 PDA

1505 PDMS

1506 PEG

1507 PEI

1508 PES

1509 PET

$1510 \quad \mathrm{PI}$

1511 PP

1512 PTFE

1513 PVA

1514 PVDF

1515 PVP-VTES

1516 RO

1517 SMM

1518 TGA

1519 TIPS

1520

WCA
Polydopamine

Polydimethylsiloxane

Polyethylene Glycol

Polyethylenimine

Polyethersulfone

Polyethylene Terephthalate

Polyimide

Polypropylene

Polytetrafluoroethylene

Polyvinyl Alcohol

Polyvinylidene Difluoride

Poly(Vinylpyrrolidone-Vinyltriethoxysilane)

Reverse osmosis

Surface Modified Macromolecules

Thermogravimetric Analysis

Temperature Induced Phase Inversion

Water Contact Angle 
1523

1524

1525

1526

1527

1528

1529

1530

1531

1532

1533

1534

1535

1536

1537

1538

1539

1540

1541

1542

1543

1544

1545

1546

1547

1548

1549

1550

1551

1552

1553

1554

1555

1556

1557

1558

1559

1560

1561

1562

1563

1564

1565

1566

1567

1568

1569

[1] A. Hosseinzadeh, J.L. Zhou, A. Altaee, M. Baziar, D. Li, Effective modelling of hydrogen and energy recovery in microbial electrolysis cell by artificial neural network and adaptive network-based fuzzy inference system, Bioresource Technology 316 (2020) 123967.

[2] C.B. Alvim, V.R. Moreira, Y.A.R. Lebron, A.V. Santos, L.C. Lange, R.P.M. Moreira, L.V.d.S. Santos, M.C.S. Amaral, Comparison of UV, UV/H2O2 and ozonation processes for the treatment of membrane distillation concentrate from surface water treatment: PhACs removal and environmental and human health risk assessment, Chemical Engineering Journal 397 (2020) 125482. [3] G. Naidu, L. Tijing, M. Johir, H. Shon, S. Vigneswaran, Hybrid membrane distillation: Resource, nutrient and energy recovery, Journal of Membrane Science 599 (2020) 117832.

[4] L.N. Nthunya, L. Gutierrez, S. Derese, E.N. Nxumalo, A.R. Verliefde, B.B. Mamba, S.D. Mhlanga, A review of nanoparticle-enhanced membrane distillation membranes: membrane synthesis and applications in water treatment, Journal of Chemical Technology \& Biotechnology 94(9) (2019) 2757 2771.

[5] A.A. Najafpoor, A. Sadeghi, H. Alidadi, M. Davoudi, B. Mohebrad, A. Hosseinzadeh, S. Jafarpour, A. Zarei, Biodegradation of high concentrations of phenol by baker's yeast in anaerobic sequencing batch reactor, Environmental Health Engineering and Management Journal 2(2) (2015) 79-86.

[6] S. Lin, Energy Efficiency of Desalination: Fundamental Insights from Intuitive Interpretation, Environmental Science \& Technology 54(1) (2019) 76-84.

[7] A. Deshmukh, C. Boo, V. Karanikola, S. Lin, A.P. Straub, T. Tong, D.M. Warsinger, M. Elimelech, Membrane distillation at the water-energy nexus: limits, opportunities, and challenges, Energy \& Environmental Science 11(5) (2018) 1177-1196.

[8] E.J. Lee, B.J. Deka, J. Guo, Y.C. Woo, H.K. Shon, A.K. An, Engineering the Re-Entrant Hierarchy and Surface Energy of PDMS-PVDF Membrane for Membrane Distillation Using a Facile and Benign Microsphere Coating, Environ Sci Technol 51(17) (2017) 10117-10126.

[9] Z. Wang, Y. Chen, S. Lin, Kinetic model for surfactant-induced pore wetting in membrane distillation, Journal of Membrane Science 564 (2018) 275-288.

[10] Z. Wang, Y. Chen, X. Sun, R. Duddu, S. Lin, Mechanism of pore wetting in membrane distillation with alcohol vs. surfactant, Journal of Membrane Science 559 (2018) 183-195.

[11] T. Horseman, Y. Yin, K.S. Christie, Z. Wang, T. Tong, S. Lin, Wetting, Scaling, and Fouling in Membrane Distillation: State-of-the-Art Insights on Fundamental Mechanisms and Mitigation Strategies, ACS ES\&T Engineering (2020).

[12] Z. Zhu, Y. Liu, H. Hou, W. Shi, F. Qu, F. Cui, W. Wang, Dual-Bioinspired Design for Constructing Membranes with Superhydrophobicity for Direct Contact Membrane Distillation, Environ Sci Technol 52(5) (2018) 3027-3036.

[13] B.J. Deka, J. Guo, N.K. Khanzada, A.K. An, Omniphobic re-entrant PVDF membrane with ZnO nanoparticles composite for desalination of low surface tension oily seawater, Water research 165 (2019) 114982.

[14] J.H. Roque-Ruiz, N.A. Medellín-Castillo, S.Y. Reyes-López, Fabrication of $\alpha$-alumina fibers by solgel and electrospinning of aluminum nitrate precursor solutions, Results in Physics 12 (2019) 193204.

[15] B.S. Chapman, S.R. Mishra, J.B. Tracy, Direct electrospinning of titania nanofibers with ethanol, Dalton Trans 48(34) (2019) 12822-12827.

[16] S. Bonyadi, T.S. Chung, Flux enhancement in membrane distillation by fabrication of dual layer hydrophilic-hydrophobic hollow fiber membranes, Journal of Membrane Science 306(1-2) (2007) 134-146.

[17] M. Khayet, T. Matsuura, Application of surface modifying macromolecules for the preparation of membranes for membrane distillation, Desalination 158(1-3) (2003) 51-56. 
[18] B.J. Deka, E.J. Lee, J. Guo, J. Kharraz, A.K. An, Electrospun Nanofiber Membranes Incorporating PDMS-Aerogel Superhydrophobic Coating with Enhanced Flux and Improved Antiwettability in Membrane Distillation, Environ Sci Technol 53(9) (2019) 4948-4958.

[19] K.J. Lu, D. Zhao, Y. Chen, J. Chang, T.-S. Chung, Rheologically controlled design of nature-inspired superhydrophobic and self-cleaning membranes for clean water production, npj Clean Water 3(1) (2020) 1-10.

[20] C. Boo, J. Lee, M. Elimelech, Omniphobic Polyvinylidene Fluoride (PVDF) Membrane for Desalination of Shale Gas Produced Water by Membrane Distillation, Environ Sci Technol 50(22) (2016) 12275-12282.

[21] C. Fornaguera, A. Dols-Perez, G. Caldero, M.J. Garcia-Celma, J. Camarasa, C. Solans, PLGA nanoparticles prepared by nano-emulsion templating using low-energy methods as efficient nanocarriers for drug delivery across the blood-brain barrier, J Control Release 211 (2015) 134-43. [22] T. Li, F. Liu, S. Zhang, H. Lin, J. Wang, C.Y. Tang, Janus Polyvinylidene Fluoride Membrane with Extremely Opposite Wetting Surfaces via One Single-Step Unidirectional Segregation Strategy, ACS Appl Mater Interfaces 10(29) (2018) 24947-24954.

[23] B. Bhushan, Y.C. Jung, Biomimetics Inspired Surfaces for Superhydrophobicity, Self-cleaning, Low Adhesion, and Drag Reduction, Nanotribology and Nanomechanics II2011, pp. 533-699. [24] X. Li, X. Yu, C. Cheng, L. Deng, M. Wang, X. Wang, Electrospun Superhydrophobic Organic/Inorganic Composite Nanofibrous Membranes for Membrane Distillation, ACS Appl Mater Interfaces 7(39) (2015) 21919-30.

[25] A. Kyoungjin An, E.J. Lee, J. Guo, S. Jeong, J.G. Lee, N. Ghaffour, Enhanced vapor transport in membrane distillation via functionalized carbon nanotubes anchored into electrospun nanofibres, Sci Rep 7 (2017) 41562.

[26] Y.C. Woo, Y. Chen, L.D. Tijing, S. Phuntsho, T. He, J.-S. Choi, S.-H. Kim, H.K. Shon, CF4 plasmamodified omniphobic electrospun nanofiber membrane for produced water brine treatment by membrane distillation, Journal of Membrane Science 529 (2017) 234-242.

[27] C. Li, X. Li, X. Du, T. Tong, T.Y. Cath, J. Lee, Antiwetting and Antifouling Janus Membrane for Desalination of Saline Oily Wastewater by Membrane Distillation, ACS Appl Mater Interfaces 11(20) (2019) 18456-18465.

[28] Y. Chen, K.J. Lu, T.-S. Chung, An omniphobic slippery membrane with simultaneous anti-wetting and anti-scaling properties for robust membrane distillation, Journal of Membrane Science 595 (2020) 117572.

[29] Z. Wang, S. Lin, Membrane fouling and wetting in membrane distillation and their mitigation by novel membranes with special wettability, Water research 112 (2017) 38-47.

[30] H.-M. Song, C. Chen, X.-X. Shui, H. Yang, L.-J. Zhu, Z.-X. Zeng, Q.-J. Xue, Asymmetric Janus membranes based on in situ mussel-inspired chemistry for efficient oil/water separation, Journal of Membrane Science 573 (2019) 126-134.

[31] M. Cao, J. Xiao, C. Yu, K. Li, L. Jiang, Hydrophobic/Hydrophilic Cooperative Janus System for Enhancement of Fog Collection, Small 11(34) (2015) 4379-84.

[32] N.G.P. Chew, S. Zhao, R. Wang, Recent advances in membrane development for treating surfactant- and oil-containing feed streams via membrane distillation, Adv Colloid Interface Sci 273 (2019) 102022.

[33] Z. Zhu, Z. Liu, L. Zhong, C. Song, W. Shi, F. Cui, W. Wang, Breathable and asymmetrically superwettable Janus membrane with robust oil-fouling resistance for durable membrane distillation, Journal of Membrane Science 563 (2018) 602-609.

[34] M. Han, T. Dong, D. Hou, J. Yao, L. Han, Carbon nanotube based Janus composite membrane of oil fouling resistance for direct contact membrane distillation, Journal of Membrane Science (2020). [35] X. Yang, L. Yan, F. Ran, A. Pal, J. Long, L. Shao, Interface-confined surface engineering constructing water-unidirectional Janus membrane, Journal of Membrane Science 576 (2019) 9-16. [36] R.Z. Waldman, H.-C. Yang, D.J. Mandia, P.F. Nealey, J.W. Elam, S.B. Darling, Janus Membranes via Diffusion-Controlled Atomic Layer Deposition, Advanced Materials Interfaces 5(15) (2018). 
[37] H.C. Yang, J. Hou, V. Chen, Z.K. Xu, Janus Membranes: Exploring Duality for Advanced Separation, Angew Chem Int Ed Engl 55(43) (2016) 13398-13407.

[38] H. Zhou, Z. Guo, Superwetting Janus membranes: focusing on unidirectional transport behaviors and multiple applications, Journal of Materials Chemistry A 7(21) (2019) 12921-12950.

[39] B. Wang, B. Li, B. Dong, B. Zhao, C.Y. Li, Homo-and hetero-particle clusters formed by janus nanoparticles with bicompartment polymer brushes, Macromolecules 43(22) (2010) 9234-9238. [40] D.Y. Cheng, S.J. Wiersma, Composite membrane for a membrane distillation system, Google Patents, 1982.

[41] D. Miao, Z. Huang, X. Wang, J. Yu, B. Ding, Continuous, spontaneous, and directional water transport in the trilayered fibrous membranes for functional moisture wicking textiles, Small 14(32) (2018) 1801527.

[42] M. Nosonovsky, B. Bhushan, Patterned nonadhesive surfaces: superhydrophobicity and wetting regime transitions, Langmuir 24(4) (2008) 1525-1533.

[43] K.J. Lu, Y. Chen, T.-S. Chung, Design of omniphobic interfaces for membrane distillation-a review, Water research 162 (2019) 64-77.

[44] J. Lee, C. Boo, W.-H. Ryu, A.D. Taylor, M. Elimelech, Development of omniphobic desalination membranes using a charged electrospun nanofiber scaffold, ACS applied materials \& interfaces 8(17) (2016) 11154-11161.

[45] P. Wang, T.-S. Chung, Recent advances in membrane distillation processes: Membrane development, configuration design and application exploring, Journal of membrane science 474 (2015) 39-56.

[46] M. Yao, L.D. Tijing, G. Naidu, S.-H. Kim, H. Matsuyama, A.G. Fane, H.K. Shon, A review of membrane wettability for the treatment of saline water deploying membrane distillation, Desalination 479 (2020) 114312.

[47] F. Huang, B. Motealleh, W. Zheng, M.T. Janish, C.B. Carter, C.J. Cornelius, Electrospinning amorphous $\mathrm{SiO} 2-\mathrm{TiO} 2$ and $\mathrm{TiO} 2$ nanofibers using sol-gel chemistry and its thermal conversion into anatase and rutile, Ceramics International 44(5) (2018) 4577-4585.

[48] Y.X. Huang, Z. Wang, J. Jin, S. Lin, Novel Janus Membrane for Membrane Distillation with Simultaneous Fouling and Wetting Resistance, Environ Sci Technol 51(22) (2017) 13304-13310. [49] M.-B. Wu, H.-C. Yang, J.-J. Wang, G.-P. Wu, Z.-K. Xu, Janus membranes with opposing surface wettability enabling oil-to-water and water-to-oil emulsification, ACS applied materials \& interfaces 9(6) (2017) 5062-5066.

[50] A.M. Pornea, J.M.C. Puguan, V.G. Deonikar, H. Kim, Robust Janus nanocomposite membrane with opposing surface wettability for selective oil-water separation, Separation and Purification Technology 236 (2020) 116297.

[51] V. Zargar, M. Asghari, M. Afsari, Gas separation properties of swelled nanocomposite chitosan membranes cross-linked by 3-aminopropyltriethoxysilane, International Journal of Environmental Science and Technology 16(1) (2019) 37-46.

[52] Z. Wang, D. Hou, S. Lin, Composite membrane with underwater-oleophobic surface for anti-oilfouling membrane distillation, Environmental science \& technology 50(7) (2016) 3866-3874.

[53] M. Li, K.J. Lu, L. Wang, X. Zhang, T.-S. Chung, Janus membranes with asymmetric wettability via a layer-by-layer coating strategy for robust membrane distillation, Journal of Membrane Science 603 (2020).

[54] L. Eykens, I. Hitsov, K. De Sitter, C. Dotremont, L. Pinoy, I. Nopens, B. Van der Bruggen, Influence of membrane thickness and process conditions on direct contact membrane distillation at different salinities, Journal of Membrane Science 498 (2016) 353-364.

[55] P.-J. Lin, M.-C. Yang, Y.-L. Li, J.-H. Chen, Prevention of surfactant wetting with agarose hydrogel layer for direct contact membrane distillation used in dyeing wastewater treatment, Journal of Membrane Science 475 (2015) 511-520.

[56] Z. Wang, Y. Chen, F. Zhang, S. Lin, Significance of surface excess concentration in the kinetics of surfactant-induced pore wetting in membrane distillation, Desalination 450 (2019) 46-53. 
[57] N.G.P. Chew, S. Zhao, C. Malde, R. Wang, Superoleophobic surface modification for robust membrane distillation performance, Journal of Membrane Science 541 (2017) 162-173.

[58] Z. Zhang, X. Du, K.H. Carlson, C.A. Robbins, T. Tong, Effective treatment of shale oil and gas produced water by membrane distillation coupled with precipitative softening and walnut shell filtration, Desalination 454 (2019) 82-90.

[59] M.B. Wu, H.C. Yang, J.J. Wang, G.P. Wu, Z.K. Xu, Janus Membranes with Opposing Surface Wettability Enabling Oil-to-Water and Water-to-Oil Emulsification, ACS Appl Mater Interfaces 9(6) (2017) 5062-5066.

[60] H.C. Yang, Y. Xie, J. Hou, A.K. Cheetham, V. Chen, S.B. Darling, Janus Membranes: Creating Asymmetry for Energy Efficiency, Adv Mater 30(43) (2018) e1801495.

[61] M. Mohammadi Ghaleni, A. Al Balushi, S. Kaviani, E. Tavakoli, M. Bavarian, S. Nejati, Fabrication of Janus Membranes for Desalination of Oil-Contaminated Saline Water, ACS Appl Mater Interfaces 10(51) (2018) 44871-44879.

[62] V. Bazrafshan, A. Saeidi, A. Mousavi, The effect of different process parameters on polyamide 66 nano fiber by force spinning method, AIP Conference Proceedings, AIP Publishing LLC, 2020, p. 020008.

[63] F. Ardeshiri, A. Akbari, M. Peyravi, M. Jahanshahi, PDADMAC/PAA semi-IPN hydrogel-coated PVDF membrane for robust anti-wetting in membrane distillation, Journal of Industrial and Engineering Chemistry 74 (2019) 14-25.

[64] J. Meng, J. Zhong, H. Xiao, J. Ou, Enhancement of strength and ductility of cement-based composites by incorporating silica nanoparticle coated polyvinylalcohol fibers, AIP Advances 9(6) (2019) 065108.

[65] J.-G. Lee, E.-J. Lee, S. Jeong, J. Guo, A.K. An, H. Guo, J. Kim, T. Leiknes, N. Ghaffour, Theoretical modeling and experimental validation of transport and separation properties of carbon nanotube electrospun membrane distillation, Journal of Membrane Science 526 (2017) 395-408.

[66] M. Afsari, M. Asghari, P. Mohammadi Moghaddam, Synthesis and characterization of supported Phenolic resin/Carbon nanotubes Carbon membranes for gas separation, International Journal of Nano Dimension 8(4) (2017) 316-328.

[67] M. Asghari, M. Afsari, Effect of Ethylene Oxide Functional Groups in PEBA-CNT Membranes on CO2/CH4 Mixed Gas Separation, Journal of Membrane Science and Research 4(1) (2018) 34-40. [68] Y. Zhao, H. Wang, H. Zhou, T. Lin, Directional Fluid Transport in Thin Porous Materials and its Functional Applications, Small 13(4) (2017).

[69] Y. Liu, T. Xiao, C. Bao, Y. Fu, X. Yang, Fabrication of novel Janus membrane by nonsolvent thermally induced phase separation (NTIPS) for enhanced performance in membrane distillation, Journal of Membrane Science 563 (2018) 298-308.

[70] M. Bazdar, A. Irankhah, Water-Gas Shift Reaction in a Microchannel Ni-based Catalytic Coated Reactor: Effect of Solvents, Chemical Engineering \& Technology (2020).

[71] L. Zou, P. Gusnawan, G. Zhang, J. Yu, Novel Janus composite hollow fiber membrane-based direct contact membrane distillation (DCMD) process for produced water desalination, Journal of Membrane Science 597 (2020) 117756.

[72] M. Asghari, M. Sheikh, M. Afsari, M. Dehghani, Molecular simulation and experimental investigation of temperature effect on chitosan-nanosilica supported mixed matrix membranes for dehydration of ethanol via pervaporation, Journal of Molecular Liquids 246 (2017) 7-16.

[73] R. Kardani, M. Asghari, N.F. Hamedani, M. Afsari, Mesoporous copper zinc bimetallic imidazolate MOF as nanofiller to improve gas separation performance of PEBA-based membranes, Journal of Industrial and Engineering Chemistry (2019).

[74] H.N. Li, J. Yang, Z.K. Xu, Asymmetric Surface Engineering for Janus Membranes, Advanced Materials Interfaces 7(7) (2020).

[75] F. Ren, G. Li, Z. Zhang, X. Zhang, H. Fan, C. Zhou, Y. Wang, Y. Zhang, C. Wang, K. Mu, Y. Su, D. $\mathrm{Wu}, \mathrm{A}$ single-layer Janus membrane with dual gradient conical micropore arrays for self-driving fog collection, Journal of Materials Chemistry A 5(35) (2017) 18403-18408. 
[76] M. Bazdar, A. Irankhah, Performance Study on Microchannel Coated Catalytic Plate Reactor Using Electrophoresis Technique for Medium Temperature Shift (MTS) Reaction, Energy \& Fuels 31(7) (2017) 7624-7634.

[77] H.-C. Yang, J. Hou, L.-S. Wan, V. Chen, Z.-K. Xu, Janus Membranes with Asymmetric Wettability for Fine Bubble Aeration, Advanced Materials Interfaces 3(9) (2016).

[78] K. Wang, D. Hou, P. Qi, K. Li, Z. Yuan, J. Wang, Development of a composite membrane with underwater-oleophobic fibrous surface for robust anti-oil-fouling membrane distillation, J Colloid Interface Sci 537 (2019) 375-383.

[79] Z. Wang, X. Yang, Z. Cheng, Y. Liu, L. Shao, L. Jiang, Simply realizing "water diode" Janus membranes for multifunctional smart applications, Materials Horizons 4(4) (2017) 701-708. [80] X. Li, W. Zhang, R. Qu, Y. Liu, Y. Wei, L. Feng, Asymmetric superwetting configuration of Janus membranes based on thiol-ene clickable silane nanospheres enabling on-demand and energyefficient oil-water remediation, Journal of Materials Chemistry A 7(16) (2019) 10047-10057. [81] Z. Wang, Y. Wang, G. Liu, Rapid and Efficient Separation of Oil from Oil-in-Water Emulsions Using a Janus Cotton Fabric, Angew Chem Int Ed Engl 55(4) (2016) 1291-4.

[82] N.G.P. Chew, Y. Zhang, K. Goh, J.S. Ho, R. Xu, R. Wang, Hierarchically Structured Janus Membrane Surfaces for Enhanced Membrane Distillation Performance, ACS Appl Mater Interfaces 11(28) (2019) 25524-25534.

[83] E.-J. Lee, A.K. An, P. Hadi, S. Lee, Y.C. Woo, H.K. Shon, Advanced multi-nozzle electrospun functionalized titanium dioxide/polyvinylidene fluoride-co-hexafluoropropylene (TiO2/PVDF-HFP) composite membranes for direct contact membrane distillation, Journal of Membrane Science 524 (2017) 712-720.

[84] L.N. Nthunya, L. Gutierrez, A.R. Verliefde, S.D. Mhlanga, Enhanced flux in direct contact membrane distillation using superhydrophobic PVDF nanofibre membranes embedded with organically modified SiO2 nanoparticles, Journal of Chemical Technology \& Biotechnology 94(9) (2019) 2826-2837.

[85] M.R.S. Kebria, A. Rahimpour, S.K. Salestan, S.F. Seyedpour, A. Jafari, F. Banisheykholeslami, N. Tavajohi Hassan Kiadeh, Hyper-branched dendritic structure modified PVDF electrospun membranes for air gap membrane distillation, Desalination 479 (2020).

[86] J.A. Kharraz, A.K. An, Patterned superhydrophobic polyvinylidene fluoride (PVDF) membranes for membrane distillation: Enhanced flux with improved fouling and wetting resistance, Journal of Membrane Science 595 (2020) 117596.

[87] S. Zhou, Z. Xiong, F. Liu, H. Lin, J. Wang, T. Li, Q. Han, Q. Fang, Novel Janus membrane with unprecedented osmosis transport performance, Journal of Materials Chemistry A 7(2) (2019) 632638.

[88] A. Vanangamudi, L.F. Dumée, M.C. Duke, X. Yang, Nanofiber composite membrane with intrinsic janus surface for reversed-protein-fouling ultrafiltration, ACS applied materials \& interfaces 9(21) (2017) 18328-18337.

[89] L. Yan, X. Yang, J. Long, X. Cheng, D. Pan, Y. Huang, L. Shao, Universal unilateral electrospinning/spraying strategy to construct water-unidirectional Janus membranes with well-tuned hierarchical micro/nanostructures, Chem Commun (Camb) 56(3) (2020) 478-481.

[90] Y. Zhang, M. Barboiu, Dynameric asymmetric membranes for directional water transport, Chemical Communications 51(88) (2015) 15925-15927.

[91] L.D. Tijing, Y.C. Woo, M.A.H. Johir, J.-S. Choi, H.K. Shon, A novel dual-layer bicomponent electrospun nanofibrous membrane for desalination by direct contact membrane distillation, Chemical Engineering Journal 256 (2014) 155-159.

[92] A. Ghaee, M. Karimi, M. Lotfi-Sarvestani, B. Sadatnia, V. Hoseinpour, Preparation of hydrophilic polycaprolactone/modified ZIF-8 nanofibers as a wound dressing using hydrophilic surface modifying macromolecules, Materials Science and Engineering: C 103 (2019) 109767. 
[93] Z.S. Tai, M.H.A. Aziz, M.H.D. Othman, A.F. Ismail, M.A. Rahman, J. Jaafar, An Overview of Membrane Distillation, Membrane Separation Principles and Applications, Elsevier2019, pp. 251281.

[94] M. Essalhi, M. Khayet, Surface segregation of fluorinated modifying macromolecule for hydrophobic/hydrophilic membrane preparation and application in air gap and direct contact membrane distillation, Journal of membrane science 417 (2012) 163-173.

[95] M. Khayet, M.C. García-Payo, L. García-Fernández, J. Contreras-Martínez, Dual-layered electrospun nanofibrous membranes for membrane distillation, Desalination 426 (2018) 174-184. [96] M. Khayet, T. Matsuura, M.R. Qtaishat, J.I. Mengual, Porous hydrophobic/hydrophilic composite membranes preparation and application in DCMD desalination at higher temperatures, Desalination 199(1-3) (2006) 180-181.

[97] X. Yue, T. Zhang, D. Yang, F. Qiu, Z. Li, Janus ZnO-cellulose/MnO2 hybrid membranes with asymmetric wettability for highly-efficient emulsion separations, Cellulose 25(10) (2018) 5951-5965. [98] A. Figoli, C. Ursino, F. Galiano, E. Di Nicolò, P. Campanelli, M.C. Carnevale, A. Criscuoli, Innovative hydrophobic coating of perfluoropolyether (PFPE) on commercial hydrophilic membranes for DCMD application, Journal of Membrane Science 522 (2017) 192-201.

[99] A.A. Najafpoor, A.J. Jafari, A. Hosseinzadeh, R.K. Jazani, H. Bargozin, Optimization of nonthermal plasma efficiency in the simultaneous elimination of benzene, toluene, ethyl-benzene, and xylene from polluted airstreams using response surface methodology, Environmental Science and Pollution Research 25(1) (2018) 233-241.

[100] A. Hosseinzadeh, A.A. Najafpoor, A.J. Jafari, R.K. Jazani, M. Baziar, H. Bargozin, F.G. Piranloo, Application of response surface methodology and artificial neural network modeling to assess nonthermal plasma efficiency in simultaneous removal of BTEX from waste gases: Effect of operating parameters and prediction performance, Process Safety and Environmental Protection 119 (2018) 261-270.

[101] J. Lee, T. Laoui, R. Karnik, Nanofluidic transport governed by the liquid/vapour interface, Nature nanotechnology 9(4) (2014) 317-323.

[102] G. Toskas, C. Cherif, R.D. Hund, E. Laourine, B. Mahltig, A. Fahmi, C. Heinemann, T. Hanke, Chitosan(PEO)/silica hybrid nanofibers as a potential biomaterial for bone regeneration, Carbohydr Polym 94(2) (2013) 713-22.

[103] H. Ye, J. Zhu, D. Deng, S. Jin, J. Li, Y. Man, Enhanced osteogenesis and angiogenesis by $\mathrm{PCL} /$ chitosan/Sr-doped calcium phosphate electrospun nanocomposite membrane for guided bone regeneration, J Biomater Sci Polym Ed 30(16) (2019) 1505-1522.

[104] Y. Chen, K.-J. Lu, S. Japip, T.-S. Chung, Can Composite Janus Membranes with an Ultrathin Dense Hydrophilic Layer Resist Wetting in Membrane Distillation?, Environmental Science \& Technology 54(19) (2020) 12713-12722.

[105] Y. Yin, W. Wang, A.K. Kota, S. Zhao, T. Tong, Elucidating mechanisms of silica scaling in membrane distillation: effects of membrane surface wettability, Environmental Science: Water Research \& Technology 5(11) (2019) 2004-2014.

[106] C. Wang, J. Wang, L. Zeng, Z. Qiao, X. Liu, H. Liu, J. Zhang, J. Ding, Fabrication of Electrospun Polymer Nanofibers with Diverse Morphologies, Molecules 24(5) (2019).

[107] H.-C. Yang, W. Zhong, J. Hou, V. Chen, Z.-K. Xu, Janus hollow fiber membrane with a musselinspired coating on the lumen surface for direct contact membrane distillation, Journal of Membrane Science 523 (2017) 1-7.

[108] L. Zou, P. Gusnawan, G. Zhang, J. Yu, Novel Janus composite hollow fiber membrane-based direct contact membrane distillation (DCMD) process for produced water desalination, Journal of Membrane Science 597 (2020).

[109] C. Su, Y. Li, H. Cao, C. Lu, Y. Li, J. Chang, F. Duan, Novel PTFE hollow fiber membrane fabricated by emulsion electrospinning and sintering for membrane distillation, Journal of Membrane Science 583 (2019) 200-208. 
[110] K.J. Lu, J. Zuo, J. Chang, H.N. Kuan, T.-S. Chung, Omniphobic hollow-fiber membranes for vacuum membrane distillation, Environmental science \& technology 52(7) (2018) 4472-4480. [111] J. Zuo, T.-S. Chung, G.S. O’Brien, W. Kosar, Hydrophobic/hydrophilic PVDF/Ultem ${ }^{\circledR}$ dual-layer hollow fiber membranes with enhanced mechanical properties for vacuum membrane distillation, Journal of Membrane Science 523 (2017) 103-110.

[112] A. Naderi, T.-S. Chung, M. Weber, C. Maletzko, High performance dual-layer hollow fiber membrane of sulfonated polyphenylsulfone/Polybenzimidazole for hydrogen purification, Journal of Membrane Science 591 (2019) 117292.

[113] E. Virga, J. de Grooth, K. Žvab, W.M. de Vos, Stable Polyelectrolyte Multilayer-Based Hollow Fiber Nanofiltration Membranes for Produced Water Treatment, ACS Applied Polymer Materials 1(8) (2019) 2230-2239.

[114] L.D. Tijing, Y.C. Woo, W.-G. Shim, T. He, J.-S. Choi, S.-H. Kim, H.K. Shon, Superhydrophobic nanofiber membrane containing carbon nanotubes for high-performance direct contact membrane distillation, Journal of Membrane Science 502 (2016) 158-170.

[115] M. Toriello, M. Afsari, H.K. Shon, L.D. Tijing, Progress on the Fabrication and Application of Electrospun Nanofiber Composites, Membranes 10(9) (2020) 204.

[116] Y.C. Woo, L.D. Tijing, M.J. Park, M. Yao, J.-S. Choi, S. Lee, S.-H. Kim, K.-J. An, H.K. Shon, Electrospun dual-layer nonwoven membrane for desalination by air gap membrane distillation, Desalination 403 (2017) 187-198.

[117] B. Yue, B. Zhang, J. You, Y. Li, L. Li, J. Li, "Lotus-effect" tape: imparting superhydrophobicity to solid materials with an electrospun Janus composite mat, RSC Advances 6(21) (2016) 17215-17221. [118] C. Boo, J. Lee, M. Elimelech, Engineering surface energy and nanostructure of microporous films for expanded membrane distillation applications, Environmental science \& technology 50(15) (2016) 8112-8119.

[119] L.D. Tijing, Y.C. Woo, M. Yao, J. Ren, H.K. Shon, 1.16 Electrospinning for Membrane Fabrication: Strategies and Applications, Comprehensive Membrane Science and Engineering2017, pp. 418-444. [120] J. Xue, T. Wu, Y. Dai, Y. Xia, Electrospinning and Electrospun Nanofibers: Methods, Materials, and Applications, Chem Rev 119(8) (2019) 5298-5415.

[121] M.R. Elmarghany, A.H. El-Shazly, S. Rajabzadeh, M.S. Salem, M.A. Shouman, M.N. Sabry, H. Matsuyama, N. Nady, Triple-Layer Nanocomposite Membrane Prepared by Electrospinning Based on Modified PES with Carbon Nanotubes for Membrane Distillation Applications, Membranes 10(1) (2020) 15.

[122] E.-J. Lee, B.J. Deka, A.K. An, Reinforced superhydrophobic membrane coated with aerogelassisted polymeric microspheres for membrane distillation, Journal of Membrane Science 573 (2019) 570-578.

[123] J.L. Wang, M. Hassan, J.W. Liu, S.H. Yu, Nanowire assemblies for flexible electronic devices: recent advances and perspectives, Advanced Materials 30(48) (2018) 1803430.

[124] J. Wu, N. Wang, L. Wang, H. Dong, Y. Zhao, L. Jiang, Unidirectional water-penetration composite fibrous film via electrospinning, Soft Matter 8(22) (2012) 5996-5999. [125] W. Sun, F. Shen, Z. Wang, Y. Zhang, Y. Wan, An ultrathin, porous and in-air hydrophilic/underwater oleophobic coating simultaneously increasing the flux and antifouling property of membrane for membrane distillation, Desalination 445 (2018) 40-50.

[126] D. Hou, Z. Wang, K. Wang, J. Wang, S. Lin, Composite membrane with electrospun multiscaletextured surface for robust oil-fouling resistance in membrane distillation, Journal of Membrane Science 546 (2018) 179-187.

[127] H. Qiu, Y. Peng, L. Ge, B.V. Hernandez, Z. Zhu, Pore channel surface modification for enhancing anti-fouling membrane distillation, Applied Surface Science 443 (2018) 217-226.

[128] D. Cheng, J. Zhang, N. Li, D. Ng, S.R. Gray, Z. Xie, Antiwettability and performance stability of a composite hydrophobic/hydrophilic dual-layer membrane in wastewater treatment by membrane distillation, Industrial \& Engineering Chemistry Research 57(28) (2018) 9313-9322. 
[129] Y. Zhan, X. Wan, S. He, Q. Yang, Y. He, Design of durable and efficient poly (arylene ether nitrile)/bioinspired polydopamine coated graphene oxide nanofibrous composite membrane for anionic dyes separation, Chemical Engineering Journal 333 (2018) 132--145.

[130] W. Xu, X. Hu, S. Zhuang, Y. Wang, X. Li, L. Zhou, S. Zhu, J. Zhu, Flexible and Salt Resistant Janus Absorbers by Electrospinning for Stable and Efficient Solar Desalination, Advanced Energy Materials 8(14) (2018). [131] L. Shi, X. Liu, W. Wang, L. Jiang, S. Wang, A Self-Pumping Dressing for Draining Excessive Biofluid around Wounds, Advanced Materials 31(5) (2019) 1804187.

[132] Y. Dong, J. Kong, C. Mu, C. Zhao, N.L. Thomas, X. Lu, Materials design towards sport textiles with low-friction and moisture-wicking dual functions, Materials \& Design 88 (2015) 82-87.

[133] C. Mendoza, Z. Gonzlez, Y. Castro, E. Gordo, B. Ferrari, Improvement of TiN nanoparticles EPD inducing steric stabilization in non-aqueous suspensions, Journal of the European Ceramic Society 36(2) (2016) 307--317.

[134] J.A. Prince, D. Rana, T. Matsuura, N. Ayyanar, T.S. Shanmugasundaram, G. Singh, Nanofiber based triple layer hydro-philic/-phobic membrane--a solution for pore wetting in membrane distillation, Sci Rep 4 (2014) 6949.

[135] C. Ursino, E. Di Nicolò, B. Gabriele, A. Criscuoli, A. Figoli, Development of a novel perfluoropolyether (PFPE) hydrophobic/hydrophilic coated membranes for water treatment, Journal of membrane science 581 (2019) 58-71.

[136] J. Kim, J. Kim, S. Hong, Recovery of water and minerals from shale gas produced water by membrane distillation crystallization, Water Res 129 (2018) 447-459.

[137] L.D. Tijing, Y.C. Woo, J.-S. Choi, S. Lee, S.-H. Kim, H.K. Shon, Fouling and its control in membrane distillation-A review, Journal of Membrane Science 475 (2015) 215-244.

[138] J.A. Bush, J. Vanneste, E.M. Gustafson, C.A. Waechter, D. Jassby, C.S. Turchi, T.Y. Cath, Prevention and management of silica scaling in membrane distillation using $\mathrm{pH}$ adjustment, Journal of Membrane Science 554 (2018) 366-377.

[139] T. Tong, A.F. Wallace, S. Zhao, Z. Wang, Mineral scaling in membrane desalination: Mechanisms, mitigation strategies, and feasibility of scaling-resistant membranes, Journal of membrane science (2019).

[140] Y. Liao, R. Wang, M. Tian, C. Qiu, A.G. Fane, Fabrication of polyvinylidene fluoride (PVDF) nanofiber membranes by electro-spinning for direct contact membrane distillation, Journal of Membrane Science 425 (2013) 30-39.

[141] Z. Xiao, R. Zheng, Y. Liu, H. He, X. Yuan, Y. Ji, D. Li, H. Yin, Y. Zhang, X.-M. Li, Slippery for scaling resistance in membrane distillation: A novel porous micropillared superhydrophobic surface, Water research 155 (2019) 152-161.

[142] Z. Xiao, Z. Li, H. Guo, Y. Liu, Y. Wang, H. Yin, X. Li, J. Song, L.D. Nghiem, T. He, Scaling mitigation in membrane distillation: From superhydrophobic to slippery, Desalination 466 (2019) 36-43. 